NISTIR 8098

\title{
Calibration and Measurement Procedures for a High Magnification Thermal Camera
}

\author{
Brandon Lane, Ph.D.
}

Eric P. Whitenton

http://dx.doi.org/10.6028/NIST.IR.8098

National Institute of Standards and Technology U.S. Department of Commerce 
NISTIR 8098

\title{
Calibration and Measurement Procedures for a High Magnification Thermal Camera
}

\author{
Brandon Lane \\ Eric Whitenton \\ Engineering Laboratory \\ Intelligent Systems Division
}

This publication is available free of charge from: http://dx.doi.org/10.6028/NIST.IR.8098

December 2015

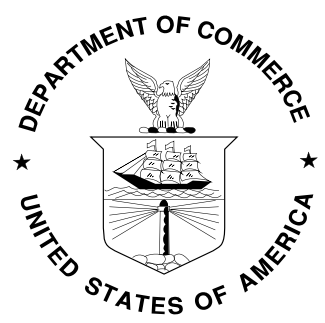

U.S. Department of Commerce Penny Pritzker, Secretary

National Institute of Standards and Technology Willie May, Acting Under Secretary of Commerce for Standards and Technology and Acting Director 
This page intentionally left blank 


\title{
Calibration and Measurement Procedures for a High Magnification Thermal Camera
}

\author{
Brandon Lane \\ Eric P. Whitenton \\ National Institute of Standards and Technology, Gaithersburg, MD USA 20899-8223
}

\begin{abstract}
Infrared thermography is a relatively mature measurement technology, however systematic evaluation of measurement uncertainty for multi-point measurement (an infrared focal plane array) is very limited, or, does not follow uncertainty evaluation guidelines outlined in the Bureau International des Poids et Mesures Guide to the Expression of Uncertainty in Measurement (BIPM-GUM). In order to approach uncertainty evaluation of thermographic measurements, a new thermographic calibration and measurement procedure was developed at the National Institute of Standards and Technology (NIST). This method takes from procedures used with single-point radiation pyrometers, which have a more developed background in uncertainty evaluation stemming from their use as calibration transfer instruments.

This report details a methodology for calibrating a thermal camera and obtaining temperature measurements, including measurement of emissivity and point spread function. This procedure is performed as a 'post-processing' technique which does not require the camera operating software to convert camera signal to temperature values. In-camera or manufacturer provided calibrations and temperature conversion algorithms may provide sufficiently low measurement uncertainty, however these results are often 'hidden' within the camera operating software and are not readily available for thorough uncertainty analysis, or, these algorithms are known but their form inhibits analytical means for uncertainty analyses.

The methodology described here is designed as a framework for future development of analytical derivations of measurement uncertainty. The detailed process also includes methods for measuring and reducing identified sources of uncertainty (e.g., point spread function) that are otherwise neglected in most thermographic measurement procedures.

Disclaimer: This document is an official contribution of the National Institute of Standards and Technology and is not subject to copyright in the United States. The National Institute of Standards and Technology does not recommend or endorse commercial equipment or materials, nor does it imply that the equipment or materials shown are necessarily the best available for the purpose.
\end{abstract}




\section{TABLE OF CONTENTS}

Abstract

Table of Contents $\quad$ ii

Table of Symbols $\quad$ iii

1 Introduction 1

2 Blackbody Calibration and Non-linearity Correction 3

2.1 Initial Imaging of the Calibration Blackbody 4

2.1.1 Calibration Blackbody Temperature Correction with Transfer Radiometer 5

2.1.2 Creating Blackbody Calibration Frames 5

2.1.3 Blackbody Imaging Results 6

$\begin{array}{lll}2.2 & \text { Non-linearity } & 8\end{array}$

2.2.1 Nonlinearity Results $\quad 9$

$\begin{array}{lll}2.3 & \text { Temperature Calibration (Sakuma-Hattori) } & 11\end{array}$

2.3.1 Sakuma-Hattori Calibration Results 13

3 Point Spread Function Measurement 15

3.1 Measuring the ESF 17

3.1.1 Measurement and Projection of the KE image 17

$\begin{array}{lll}3.2 & \text { Formation of the 3D PSF } & 23\end{array}$

3.3 PSF Deconvolution and Results 23

4 Emissivity Measurement 27

4.1 Calculating Emissivity 30

4.2 Emissivity Measurement Results 31

5 True Temperature Measurement $\quad 32$

6 Discussion $\quad 34$

7 Conclusions $\quad 35$

$\begin{array}{ll}\text { References } & 37\end{array}$

Appendix A. Notation and Nomenclature for Image Statistics .................................. A-1

Appendix B. Spectral Responsivity and Sakuma-Hattori Equation Relationships .............. B-1

Appendix C. Calibration Data Structure and Functions .................................................. C-1 


\section{TABLE OF SYMBOLS}

\begin{tabular}{|c|c|}
\hline Symbol & Name and units \\
\hline$T_{b b}$ & True blackbody temperature $\left[{ }^{\circ} \mathrm{C}\right]$ \\
\hline$T_{b b, T C}$ & Calibration blackbody thermocouple temperature $\left[{ }^{\circ} \mathrm{C}\right]$ \\
\hline$T_{b b, r a d}$ & Calibration blackbody radiant temperature $\left[{ }^{\circ} \mathrm{C}\right]$ \\
\hline$T_{T C}$ & Thermocouple temperature (during emissivity measurement) $\left[{ }^{\circ} \mathrm{C}\right]$ \\
\hline$T_{a p p}$ & Apparent or radiometric temperature $\left[{ }^{\circ} \mathrm{C}\right]$ \\
\hline$S_{\mu}$ & Signal (during emissivity measurement) [a.u.] \\
\hline$S_{m B B}$ & Average signal from micro-blackbody area [a.u.] \\
\hline$S_{\text {meas }}$ & Signal image of a measured scene [a.u.] \\
\hline$\eta(S)$ & Linearization function \\
\hline$\eta_{b b}$ & Linearization function evaluated at blackbody calibration points \\
\hline$a, b, c, k$ & Linearization function coefficients \\
\hline$F(S)$ & Calibration equation (Sakuma-Hattori) \\
\hline$A, B, C$ & Sakuma-Hattori equation coefficients \\
\hline$c_{1 L}$ & First radiation constant $\left(1.1910439 \times 10^{-16}\left[\mathrm{~W} \cdot \mathrm{m}^{2}\right]\right)$ \\
\hline$c_{2}$ & Second radiation constant $(0.014338[\mathrm{~m} \cdot \mathrm{K}])$ \\
\hline$\lambda$ & Wavelength $[\mu \mathrm{m}]$ \\
\hline$\tau_{\text {lens }}(\lambda), \tau_{\text {filter }}(\lambda)$. & Relative spectral transmission of lens or filter [-] \\
\hline$R(\lambda)$ & Relative responsivity of camera (sans optic components) [-] \\
\hline$w_{\lambda}$ & Relative responsivity of camera system (including filters and optics) [-] \\
\hline$\mu F()$ & Frame mean; mean of every pixel value in one frame [various] \\
\hline$\sigma F()$ & Frame noise; standard deviation of every pixel value in one frame \\
\hline$\mu P()$ & Pixel mean; mean of individual pixel value over multiple frames \\
\hline$\mu P()$ & Pixel noise; standard deviation of individual pixel value over multiple frames \\
\hline$\epsilon$ & Emissivity $[-]$ \\
\hline$\Delta \epsilon$ & Difference in emissivity between two temperatures [-] \\
\hline$\epsilon_{m B B}$ & Micro-blackbody emissivity [-] \\
\hline$\phi_{b b}$ & Radiant flux from a blackbody source [W] \\
\hline$L_{\lambda}(T)$ & Spectral radiance $\left[\mathrm{W} / \mathrm{sr} / \mathrm{m}^{3}\right]$ \\
\hline
\end{tabular}




\section{INTRODUCTION}

The procedures outlined in this document are an extension and improvement on the authors' previous work in uncertainty analysis for infrared thermography [1,2]. Targeted modifications to the equipment and calibration and measurement processes were made in order to reduce or quantify measurement uncertainty. Infrared thermography is a complicated measurement process, making it apt for Monte-Carlo simulations for uncertainty analysis [1,3]. As pointed out in [1], some components of uncertainty may be determined analytically, while more complicated factors likely require numerical simulation. Analytical solutions provide the benefit of yielding analytical equations which address the complex interrelationships between process variables and components of uncertainty. However, the complexities of some sources of uncertainty inhibit analytical methods. Monte-Carlo methods allow all components of uncertainty to be evaluated by iteratively reproducing the measurement process chain with input parameters specified by some probability density functions. While the Monte-Carlo method can calculate certain uncertainty component values which analytical methods cannot, they generally yield only statistical correlations which can miss details of input parameter and measurement uncertainty relationships. In addition to improvements to the physical thermographic measurement system, improvements to the calibration and measurement process were made to facilitate analytical methods for calculating measurement uncertainty and reduce measurement uncertainty overall.

This report describes the new calibration and measurement process, and presents the foundation for a more thorough approach to the measurement uncertainty than in [1]. This report details only the calibration and measurement procedures, but does not show how the measurement uncertainty is calculated. These procedures are part of a newly developed measurement process chain shown in the schematic in Figure 1 and detailed throughout this paper. Commercial thermal camera software enables users to use factory calibrations and internal, sometimes proprietary calculations, making uncertainty evaluation difficult. To fully understand and analyze the process, each step in Figure 1 uses custom-developed pre- or post-processing software. The subsequent chapters in this document are organized according to this diagram. In developing this new calibration and measurement procedure, four new components are introduced:

1.) Nonlinearity measurement and correction using an analytical model applied on a pixelby-pixel basis

2.) Blackbody calibration using an analytical model applied on a pixel-by-pixel basis (Sakuma-Hattori equation)

3.) Measurement of the spatial resolution and the point spread function (PSF)

4.) Measurement and calculation of emissivity 
1.) Non-linearity

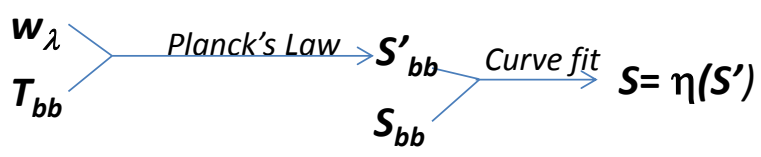

\section{2.) Blackbody Calibration}

$$
S_{b b}^{\prime} \stackrel{\eta}{\longrightarrow} \underset{T_{b b}}{S_{b b}}>\text { curve fit } \rightarrow S=F\left(T_{b b}\right)
$$

\section{3.) PSF Measurement}

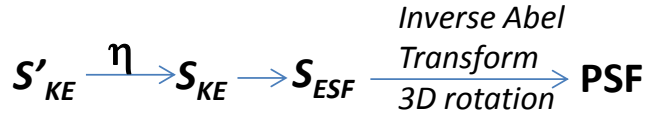

\section{4.) Emissivity Measurement}

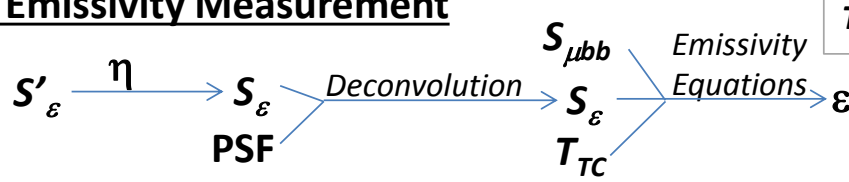

$\boldsymbol{w}_{\lambda}-$ spectral responsivity

$T_{b b}$ - calibration blackbody temperature $\left({ }^{\circ} \mathrm{C}\right)$

$\mathbf{S}^{\prime}, \mathbf{S}-$ raw and linearized signal $(\Delta \mathrm{DL})$

$S_{b b}-$ signal at calibration points $(\Delta \mathrm{DL})$

$\eta$ - Linearization function

$\mathbf{F}\left(\boldsymbol{T}_{\text {app }}\right)$ - calibration equation (Sakuma-Hattori)

$S_{K E}-$ knife edge image (a.u.)

$\boldsymbol{S}_{E S F}$ - edge spread function (a.u.)

PSF - point spread function (a.u.)

$\boldsymbol{S}_{\varepsilon}$ - emissivity signal image (a.u.)

$\varepsilon$ - object emissivity value

$\boldsymbol{S}_{\mu b b}$ - emissivity signal image (a.u.)

$T_{T C}$ - thermocouple indicated temperature $\left({ }^{\circ} \mathrm{C}\right)$

$T_{a m b}$ - reflected ambient temperature $\left({ }^{\circ} \mathrm{C}\right)$

$T_{\text {true }}$-true measured object temperature $\left({ }^{\circ} \mathrm{C}\right)$

$T_{a p p}$-apparent or radiometric temperature $\left({ }^{\circ} \mathrm{C}\right)$

\section{5.) Object Measurement}

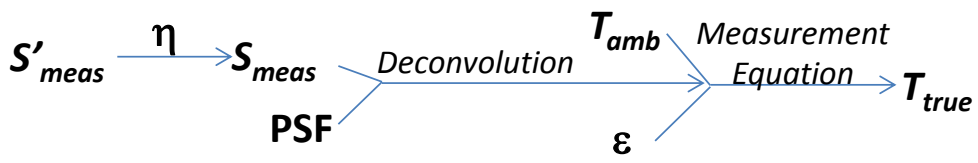

Figure 1: Outline of the calibration and measurement procedure.

The example camera system and results in this document are for a modified commercial off-theshelf (MOTS) infrared camera shown in Figure 2. Modifications include water-cooled chill plates and insulating foam around the camera body which keep the camera body temperature at $20.52{ }^{\circ} \mathrm{C}\left( \pm 0.28{ }^{\circ} \mathrm{C}, \mathrm{k}=2.2\right.$ for $95 \%$ confidence $)$ measured 11 times via internal thermistor over 4 hours, and $85 \mathrm{~mm}$ extension tube with $5 \mathrm{x}$ near infrared (NIR) microscope objective. Though not explicitly stated in [1], measurement uncertainty due to emissivity may be substantially decreased for NIR as opposed to the mid-wave infrared (MIR) systems for higher temperature (> $500{ }^{\circ} \mathrm{C}$ ) measurements since sensor signal values increase more rapidly with temperature as wavelength shortens. The objective also has a conical shroud made of brazed sheet brass, covered in fiberglass tape, and terminating in a bakelite standoff. This light cone blocks ambient sources and reflections from entering the objective lens while protecting it from stray hot chips commonly encountered in metal-cutting experiments. Multiple calibrations were performed for different filter and optics options and integration times, however only one is detailed here. All examples in this report are for the camera system shown in Figure 2. 


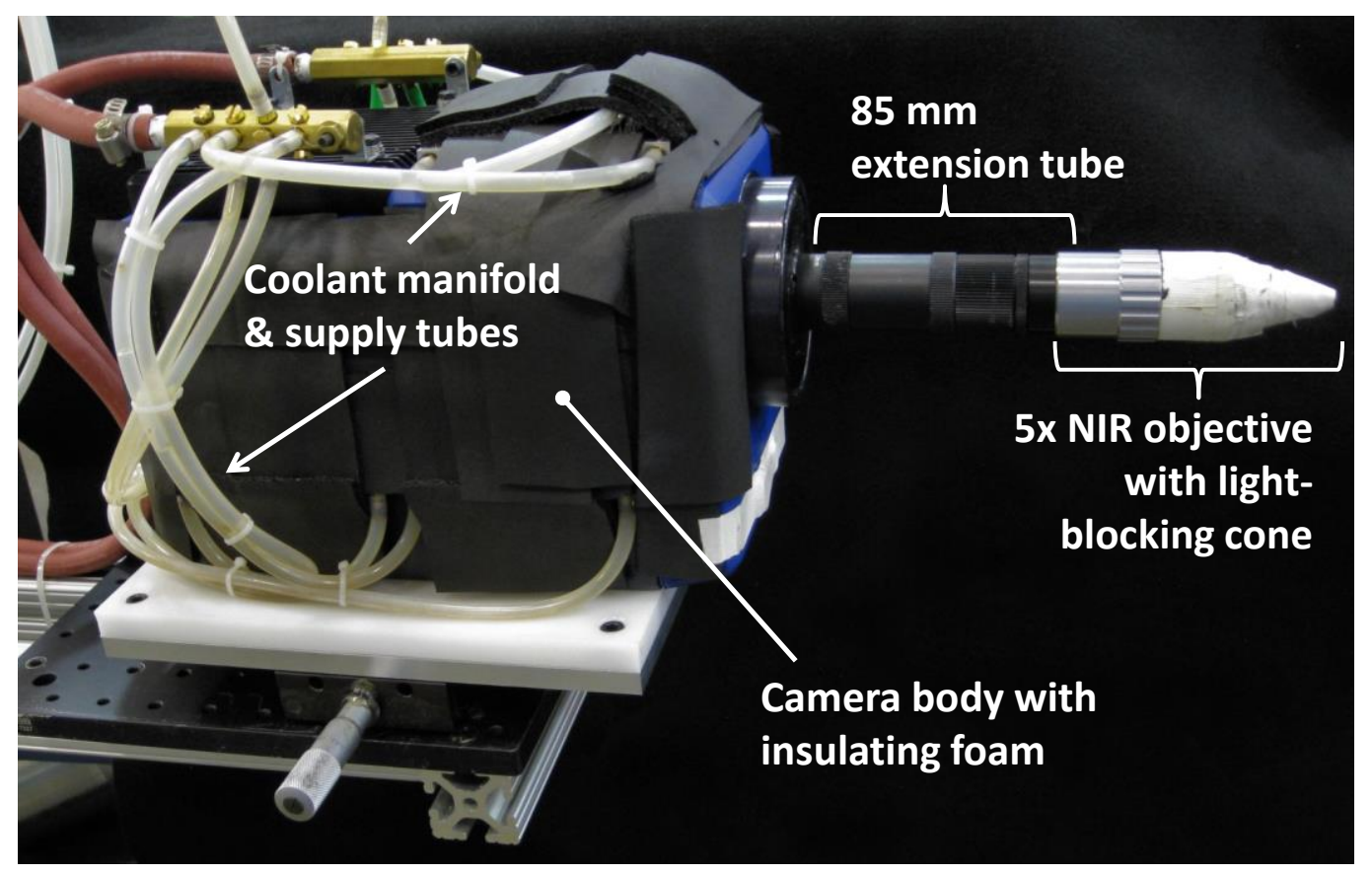

Figure 2: Modified commercial off-the-shelf (MOTS) camera used in example calibration and measurements

Images are windowed to 256 pixels x 320 pixels. This set of optics gives the MOTS camera a field of view (FoV) of $1.10 \mathrm{~mm}$ by $1.38 \mathrm{~mm}$, and a $3.365 \mu \mathrm{m} /$ pixel instantaneous field of view (iFoV). This results in 4.46x magnification on the $15 \mu \mathrm{m} /$ pixel focal plane array (FPA).

\section{BLACKBODY CALIBRATION AND NON-LINEARITY CORRECTION}

For some calculations and results comparisons, the camera spectral responsivity is required. For the MOTS camera, spectral responsivity is calculated by taking relative spectral responsivity of the camera and relative spectral transmission curves for the microscope objective and filter from manufacturer's specifications. These curves, shown in Figure 3, are copied and digitized to 10 $\mathrm{nm}$ intervals, then multiplied together to form the system relative spectral responsivity, $w_{\lambda}$. Use of the relative spectral responsivity does not require any particular scaling as $w_{\lambda}$ is typically scaled to unity in this report.

$$
w_{\lambda}=\tau_{\text {lens }}(\lambda) \cdot \tau_{\text {filter }}(\lambda) \cdot R_{\text {camera }}(\lambda)
$$




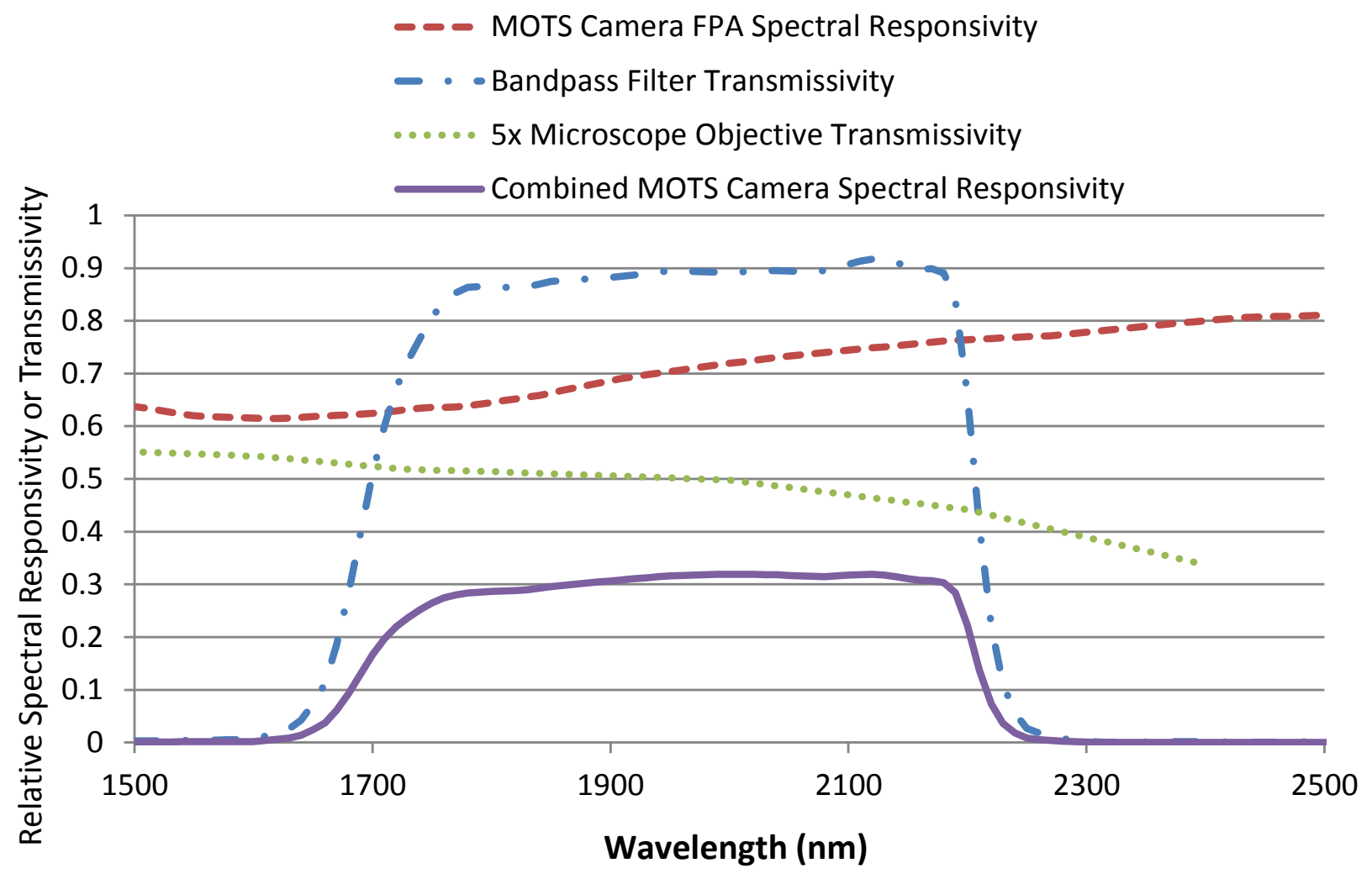

Figure 3: Combined spectral responsiviy of the MOTS camera determined from spectral responsivity/transmissivity of each component.

For this paper, the manufacturer's specification for spectral responsivity is assumed accurate, though more accurate measurement methods are available. For example, several works provide measurement uncertainty for measuring spectral uncertainties $[4,5])$.

\subsection{Initial Imaging of the Calibration Blackbody}

Before any temperature measurements are made, the response of the camera FPA needs to be related to a thermodynamic temperature. This is done by imaging a commercial, variable temperature, spherical cavity calibration blackbody. The term calibration blackbody is used throughout this paper to distinguish the instrument used to calibrate radiometric devices from the physics definition of a blackbody (i.e., a perfect radiator). The calibration blackbody provides a nominally uniform emittance scene with spectral dependence closely resembling that of a perfect radiator. This essentially ties the thermal camera signal, through Planck's radiation law, to a precisely known temperature. In reality, indicated temperature of the instrument does not perfectly relate to the exitance from the blackbody cavity. This error may be partially corrected by cross-calibrating with a transfer radiometer, which in turn is calibrated based on temperature defined by the International Temperature Scale of 1990 [6]. 


\subsubsection{Calibration Blackbody Temperature Correction with Transfer Radiometer}

The calibration blackbody was measured using the NIST Advanced Pyrometer Transfer Radiometer (APXR) traceable to the gold point $\left(1064.18{ }^{\circ} \mathrm{C}\right)$ using the $650 \mathrm{~nm}$ narrowband filter. This wavelength is outside the sensitivity band of the thermal camera in Figure 3, which may incur error if the calibration blackbody emissivity is different at these different wavelength ranges. However, spectral dependence of the calibration blackbody's non-unity emissivity is assumed to be negligible. The calibration blackbody setpoint temperature (measured via internal thermocouple) is compared to radiometric temperature value output by the APXR, and a regression curve was fit to the error at 5 blackbody set-points between $599{ }^{\circ} \mathrm{C}$ to $1000{ }^{\circ} \mathrm{C}$. This regression resulted in a standard error of estimate (SEE) of $0.25{ }^{\circ} \mathrm{C}$. This error formula is given as follows:

$$
E=\left(T_{b b, T C}-T_{A P X R}\right)=4.384 \ln \left(T_{b b, T C} /{ }^{\circ} C\right)-27.334 \quad \text { for } \quad T_{b b, T C}>510.3^{\circ} \mathrm{C}
$$

The error value is subtracted from the calibration blackbody thermocouple reading, $T_{b b, T C}$, to give radiant temperature of the blackbody $T_{b b, r a d}=T_{b b, T C}-E$. The calibration blackbody has a known effective emissivity of $\varepsilon_{b b} \leq 0.995$, therefore the radiant temperature must be converted to a true thermodynamic temperature, $T_{b b}$. This is accomplished using the spectral measurement equation and numerical minimization routine detailed in [1] and [2] to solve for $T_{b b}$.

$$
\int_{\lambda_{1}}^{\lambda_{2}} \frac{w_{\lambda}}{\lambda^{5}}\left[\frac{1}{\exp \left(c_{2} / \lambda T_{b b, r a d}\right)-1}\right] d \lambda=\int_{\lambda_{1}}^{\lambda_{2}} \frac{w_{\lambda}}{\lambda^{5}}\left[\frac{\varepsilon_{b b}}{\exp \left(c_{2} / \lambda T_{b b}\right)-1}+\frac{1-\varepsilon_{b b}}{\exp \left(c_{2} / \lambda T_{a m b}\right)-1}\right] d \lambda
$$

Note that temperatures in (3) are in Kelvin. Bandwidth limits $\lambda_{1}$ and $\lambda_{2}$ are $1.5 \mu \mathrm{m}$ and $2.5 \mu \mathrm{m}$, respectively, and ambient reflected temperature, $T_{a m b}$, is assumed to be $20{ }^{\circ} \mathrm{C}$. Bandwidth limits are not strictly chosen, but set such that the integration limits are well within the zero-value range of the camera relative spectral responsivity. Results for the calibrated conversion from setpoint to radiant to true blackbody temperature are given in Table 1.

\subsubsection{Creating Blackbody Calibration Frames}

When acquiring thermal images, researchers generally desire real-time images that are as clear as possible. All FPAs have inherent non-uniform sensitivity from pixel to pixel, which results in non-uniform or 'stripy' raw signal images. In addition, vignetting from the optics may cause pixels around the periphery of the FoV to appear darker. Thus, an in-camera non-uniformity correction (NUC) is typically used even if the images will be post-processed (as they are done in this report). A NUC assigns unique gain and offset values to each pixel so that the signal across the FPA's pixels appears uniform when a truly uniform scene is imaged. A NUC may be applied to the pixel raw signal on the FPA chip (in-camera NUC), or through software after image data is acquired (software NUC), or both.

Before any calibration is performed, a single-point in-camera NUC is performed for each integration time used and stored on the hard drive. Whether calibrating or measuring a 
temperature, an integration time is first selected and the associated in-camera NUC is recalled from the hard drive. Since the same in-camera NUC is always used for that integration time, the calibration and subsequent temperature measurements will be valid.

If an unchanging scene is imaged at two different times, resulting camera signal may change due to systematic drift. By subtracting a standard uniform thermal scene from a target measurement scene, systematic drift is canceled (except for any drift that occurs during the time between the target measurement and standard scene measurement). A standard thermal scene is created by imaging an emissivity plate (e-plate); an aluminum heat exchanger plate connected in-line with the camera liquid cooling system that is painted on one side with high emissivity black paint. The other side is a diffusely reflective, as-manufactured aluminum surface. The liquid cooling system maintains the e-plate between $12.8{ }^{\circ} \mathrm{C}$ and $13.9{ }^{\circ} \mathrm{C}$. The plate is held directly in front of the camera with the black side facing the camera and aluminum side facing the blackbody, and 100 frames are collected immediately before or after a measurement. These 100 frames are then averaged and subtracted from the corresponding calibration frames, or frames taken during an actual measurement.

Each time the calibration blackbody is imaged, the e-plate is imaged immediately before or after. At each setpoint temperature, the calibration blackbody is allowed at least an hour to reach equilibrium, reaching the setpoint to within $0.1{ }^{\circ} \mathrm{C}$ (resolution of the blackbody digital readout), and 100 frames are collected. The e-plate is measured and 100 frames collected either immediately before or after the blackbody measurement. The e-plate measurement in digital levels (DLs) are subtracted from the blackbody measurement in DLs, which are then expressed here as unit $\triangle \mathrm{DLs}$ to indicated a subtraction has been made.

A bad pixel list is manually created after all blackbody setpoints are measured by visually inspecting the calibration frames, and noting the location of unresponsive pixels. The 100 eplate frames at each setpoint are averaged and subtracted from the averaged 100 blackbody frames to create $\Delta \mathrm{DL}$ data frames at each setpoint temperature. These data frames are plotted, and outlier pixels are manually selected and their pixel coordinates noted. Outliers are visually determined since their deviation from the frame mean is obvious and likely more than $10 \sigma$ from the frame mean. Since calibration is conducted on a pixel-by-pixel basis, even pixels with responses significantly different than the mean response (e.g., more than $3 \sigma$ from calibration frame means) may be utilized.

\subsubsection{Blackbody Imaging Results}

In this example, 12 total calibration points were measured. Table 1 gives the calibration blackbody temperatures at each setpoint and the corrected values using Equations (2) and (3). Each calibration frame image is the mean of a 100-frame blackbody measurement (resulting in a single frame) minus the mean of a 100-frame e-plate measurement (also resulting in a single frame). Raw signal of either the blackbody or e-plate measurements is in units of digital levels (DL). After the e-plate measurement is subtracted, signal values are noted with units of $\Delta \mathrm{DL}$. 
Rather than show each of the 12 resulting calibration frames, results given in Table 1 give the frame mean (mean of all pixel values from 256 pixel x 320 pixel frame) of the pixel mean (pixelby-pixel mean of 100 frames). That is, 100 frames are averaged to form one frame, then all the pixels in that frame are averaged. The frame noise is also given, calculated as to standard deviation of a 256 pixel x 320 pixel frame. The frame noise gives a measure of non-uniformity, or pixel-to-pixel variation in a frame. The 12 calibration frames are stored as 256 pixel x 320 pixel frames in the calibration data structure. Further explanation of frame mean, frame noise, pixel mean, pixel noise, and how calibration frames are created is given in Appendix A.

Table 1: Blackbody measurement results for the example calibration set. Note that bad pixels are excluded from these calculations.

\begin{tabular}{|c|c|c|c|c|}
\hline \multicolumn{3}{|c|}{$\begin{array}{c}\text { Calibration Blackbody } \\
\text { Temperature }\end{array}$} & \multicolumn{2}{|c|}{ Calibration frame statistics } \\
\hline $\begin{array}{c}\text { Set-point } \\
\mathbf{T}_{\text {bb,TC }} \\
{\left[{ }^{\circ} \mathrm{C}\right]}\end{array}$ & $\begin{array}{c}\text { Radiant } \\
\mathbf{T}_{\mathbf{b b}, \mathbf{r a d}} \\
{\left[{ }^{\circ} \mathrm{C}\right]}\end{array}$ & $\begin{array}{c}\text { True } \\
\mathbf{T}_{\mathbf{b b}} \\
{\left[{ }^{\circ} \mathrm{C}\right]}\end{array}$ & $\begin{array}{c}\text { Frame mean of } \\
\text { the pixel mean } \\
\boldsymbol{\mu} \mathbf{F}(\boldsymbol{\mu} \mathrm{P}) \\
{[\Delta \mathrm{DL}]}\end{array}$ & $\begin{array}{c}\text { Frame noise } \\
\text { of pixel mean } \\
\boldsymbol{\sigma} \mathbf{F}(\boldsymbol{\mu} \mathbf{P}) \\
{[\Delta \mathrm{DL}]}\end{array}$ \\
\hline 300 & 300 & 299.8 & 25.26 & 0.66 \\
\hline 350 & 350 & 349.7 & 68.50 & 0.89 \\
\hline 400 & 400 & 399.7 & 158.57 & 1.76 \\
\hline 450 & 450 & 449.6 & 326.49 & 3.08 \\
\hline 500 & 500 & 499.6 & 619.42 & 5.64 \\
\hline 550 & 549.7 & 549.2 & 1089.84 & 9.62 \\
\hline 600 & 599.3 & 598.8 & 1797.35 & 15.13 \\
\hline 650 & 648.9 & 648.4 & 2795.67 & 28.06 \\
\hline 700 & 698.6 & 698.0 & 4155.31 & 35.11 \\
\hline 750 & 748.3 & 747.6 & 5941.68 & 51.31 \\
\hline 800 & 798.0 & 797.2 & 8269.20 & 68.31 \\
\hline 840 & 837.8 & 837.0 & 10487.61 & 85.65 \\
\hline
\end{tabular}

The frame noise at each calibration point was typically $0.8 \%$ of the frame mean. The frame mean of the pixel mean indicates that on average, a thermal image of a $300{ }^{\circ} \mathrm{C}$ blackbody will result in roughly 25 more DLs than the e-plate measurement. E-plate measurements ranged from frame means of 1687.80 DL to 1737.71 DL, with frame noise of pixel mean ranging from 0.92 DL to 21.60 DL. Though it contributes negligibly to the calibration (assuming noise levels were symmetric about the mean), it was noticed that noise levels for some e-plate measurements were appreciably higher for some measurements. It was determined that this occurred when e-plate measurements were taken immediately after a blackbody measurement. It is possible that slight heating of the FPA may have occurred in the short time it was exposed to the blackbody, which may have influenced the baseline noise of the e-plate measurement. As a result, all future calibrations will ensure e-plate measurements are taken before the blackbody. 


\subsection{Non-linearity}

In most commercial thermal camera systems, raw camera signal is scaled by the in-camera NUC, then this signal is related to calibration blackbody temperature; most often through linear interpolation, though spline interpolation, polynomial, or other curve fitting may be used. This is relatively simple, sufficient for most measurement applications, often built into commercial camera software, and often calibrated by the camera manufacturer. For most commercial cameras, raw pixel signal is very linear with incident radiant flux, therefore it is presumed that measured signal is directly related to blackbody temperature through Planck's law. However, we choose to separate the signal-to-flux and the signal-to-temperature relationships, which will later show how the MOTS camera response is slightly non-linear. By using multiple steps (linearization curve-fitting and then calibration-curve fitting), 'linearized' camera signal values are available that are directly related to flux without the assumption of linearity. This provides several benefits: 1) Measurement of the nonlinearity provides insight into the optimal measurement range of the camera (e.g., if the measured range is near the upper or lower limits of the camera's dynamic range) and 2) Calculation of point spread function (PSF) or emissivity from measured signals may incur error if these signal values are in the non-linear ranges of the camera.

For an incremental change in incident radiant flux on the FPA, the camera will give an incremental change in signal. Linearity describes how constant these incremental changes are over the measurement range of the camera. In other words, if incident flux is plotted vs. signal, it describes how close the data follows a straight line. This ties a camera signal to a radiant flux, which is related to the thermodynamic temperature of the target. Linearity can be measured using standard flux sources [7], or based on a calculated flux, which is done here by starting with Planck's equation:

$$
L_{\lambda}(T)=\frac{c_{1 L}}{\lambda^{5}\left[\exp \left(c_{2} / \lambda T\right)-1\right]}
$$

Integrated over the spectral sensitivity of the camera, the resultant temperature-dependent value is proportional to the radiance of the emitting surface. For a hypothetical perfectly linear camera, the measured signal $S$ will be proportional to the incident radiant flux on the detector $\phi$, and thus proportional to the emitted radiant flux of the blackbody.

$$
S \propto \phi=\alpha \cdot \int w_{\lambda} L_{\lambda}(T) d \lambda+\beta
$$

Here, $\alpha$ is a constant of proportionality that depends on electronics amplification, pixel size, optics geometry and transmission [8], etc. and $\beta$ is an offset representing the noise floor. Using this hypothetical linear signal calculated using equation (5) for each calibration point, a signal from a non-linear camera can be converted into values (in arbitrary units, or [a.u.]) that are 
linearly related to incident flux on the FPA by using a linearization function, $\eta$. The linearization function is calculated in equation (6.1). Equation (6.1) says that the function $\eta$ maps the ratio of calibration points $\left(S_{b b}\right.$, or nonlinear signal in $\left.[\Delta \mathrm{DL}]\right)$ over the hypothetical linear signal $\left(\phi_{b b}\right)$ back to the calibration point. This mapping is realized by fitting a function, equation (6.2), between $\eta_{b b}$ ( $\eta$ calculated at the calibration points) and $S_{b b}$ (signal measured while imaging the blackbody). Once the fit function is developed, equation (6.3) is used to convert any collected non-linear signal $S^{\prime}[\Delta \mathrm{DLs}$ ] into a linearized form $\mathrm{S}$ [a.u.].

$$
\begin{gathered}
\eta: \frac{\phi_{b b}}{S_{b b}} \rightarrow S_{b b} \\
\eta\left(S^{\prime}\right)=k\left[1-\left(a+b\left(S^{\prime}\right)^{c}\right)\right] \\
S=\eta\left(S^{\prime}\right) \cdot S^{\prime}
\end{gathered}
$$

The linearity fit equation in (6.2) is the same used in [7], although any functional form may be used, and will depend on how $\phi_{b b}$ is scaled. The real response of a thermal camera is typically Sshaped, with a linear response limited on the low end by electronic noise and saturated at the upper end [8]. If calibration points are chosen that reach far enough outside the nominally linear response, a different linearization function than (6.2) may be appropriate. The scaling parameter $\alpha$ in equation (5) was arbitrarily chosen such that $\phi$ was nominally equal to $S^{\prime}$ at the highest calibration point (resulting in $\eta \approx 1$ ). This ensured (6.2) is an appropriate function to describe $\eta$.

If applied individually to each pixel in an image, linearizing acts as a non-uniformity correction. Generally, an in-camera NUC assumes linearity. That is, if a NUC is performed at one uniform temperature scene, capturing other uniform temperature scenes will also result in uniform images. In reality, the inherently different non-linearity of each pixel will result in a nonuniform image. By linearizing each pixel individually, this acts as non-uniformity correction beyond in-camera NUC taken before the blackbody measurement. This inherent NUC caused by linearization is limited by the uniformity of the original calibration frame and goodness of fit of the curve-fit for each pixel.

\subsubsection{Nonlinearity Results}

For each pixel, $\eta$ was calculated from the 12 calibration points using equation (6.1) and a leastsquares fit used equation (6.2) to generate the coefficients of $\eta(S)$. Example fit results are given for three pixels in Figure 4 (left). Four new 256 pixel x 320 pixel arrays/frames composed of the linearization coefficient values $k, a, b$, and $c$ for each pixel are created and stored in the calibration data structure along with fit statistics. Using these linearization coefficient frames, the calibration frames $\left(S_{b b}\right)$ were linearized and converted to [a.u.]. Figure 4 (right) gives the frame mean and frame noise of the linearized calibration frames. This shows the relative range of the values of $\eta$ over the FPA. 

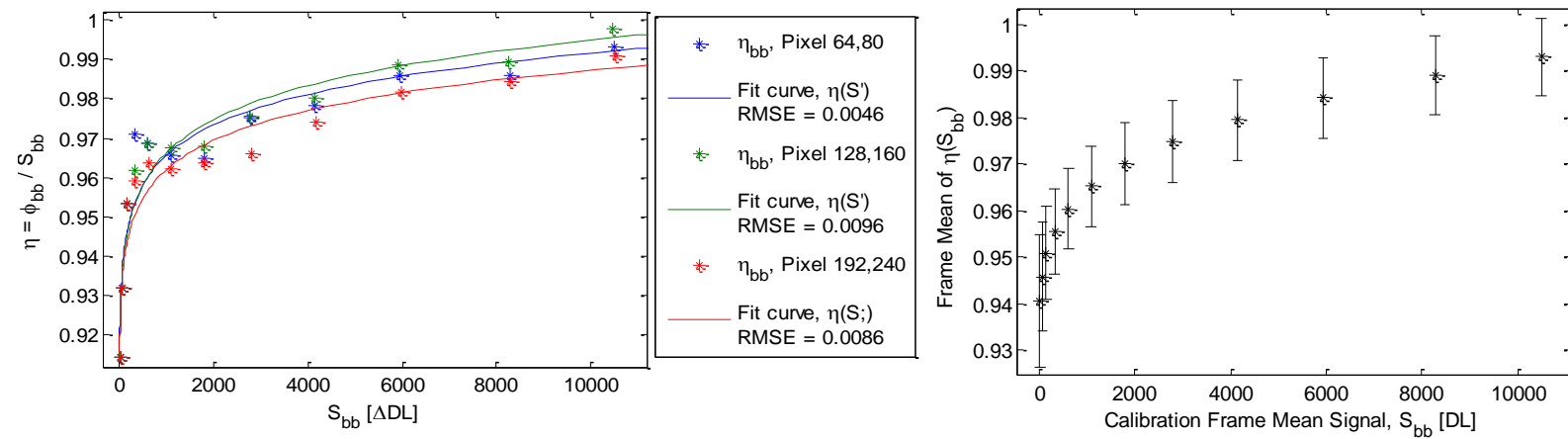

Figure 4: Left: Example nonlinearity $\eta[$ a.u. / $\Delta \mathrm{DL}]$ and corresponding fit functions for 12 calibration points and three example pixels. Right: Frame mean of $\eta$ for each of the 12 linearized calibration frames. Error bars are one standard deviation (frame noise).

Table 2 gives the frame mean and noise for the four linearization coefficient frames, and two fit statistics frames. Each pixel has its own set of coefficients and fit statistics, and Table 2 gives mean values of all pixels. Fit statistics include the coefficient of determination $\left(r^{2}\right)$ and standard error of the estimate (SEE).

Table 2: Frame mean and frame noise of 256 pixel x 320 pixel frames consisting of each pixel's linearization fit coefficients $(a, b, c$, and $k)$ and fit statistics $\left(r^{2}\right.$ and SEE)

\begin{tabular}{ccc}
$\begin{array}{c}\text { Linearization } \\
\text { Coefficient and Fit } \\
\text { Statistics }\end{array}$ & $\begin{array}{c}\text { Frame Mean } \\
\mu \mathrm{F}()\end{array}$ & $\begin{array}{c}\text { Frame Noise } \\
\sigma \mathrm{F}()\end{array}$ \\
\hline$a$ & 0.8762 & 0.3762 \\
$b$ & 0.2584 & 0.4281 \\
$c$ & 0.2788 & 0.2494 \\
$k$ & 0.4364 & 0.0366 \\
$r^{2}$ & 0.860 & 0.1166 \\
SEE & 0.0078 & 0.0029
\end{tabular}

As mentioned, the linearization process acts as a post-processing NUC. Figure 5 and Figure 6 show how two example calibration frames become more uniform after linearization. Apart from the visibly diminished vignetting, frame noise and range diminish through linearization. 

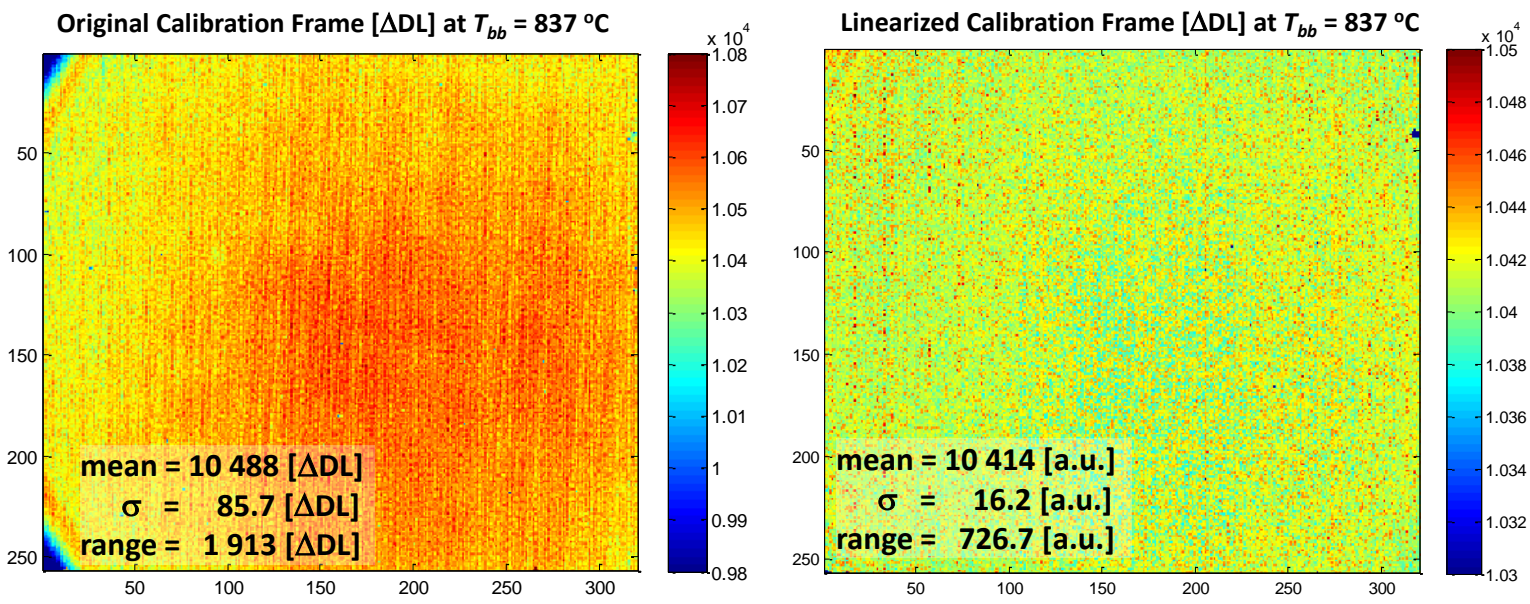

Figure 5: Comparison of a $T_{b b}=837{ }^{\circ} \mathrm{C}$ raw blackbody image (minus e-plate measurement) (left) and the results after being linearized (right) showing reduced non-uniformity as a result of the linearization process.
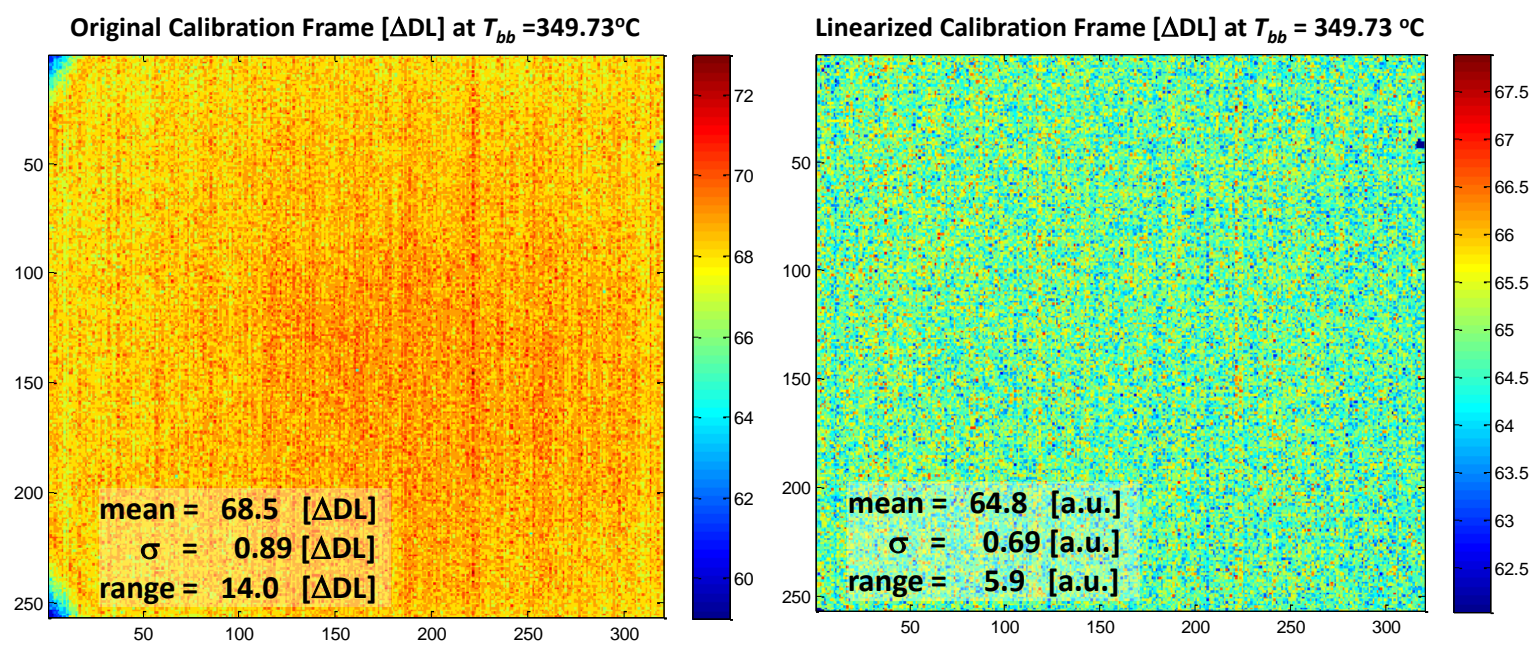

Figure 6: Comparison of a $T_{b b}=349.7^{\circ} \mathrm{C}$ raw blackbody image (minus e-plate measurement) (left) and the results after being linearized (right) showing reduced non-uniformity as a result of the linearization process.

While linearization converts raw camera signal into values linearly proportional to the radiant flux on the FPA, a further radiometric calibration is required in order to convert linearized signal to a temperature.

\subsection{Temperature Calibration (Sakuma-Hattori)}

Equation (7) describes a nonlinear regression fit done for each pixel that relates a measured signal $S$ to true blackbody temperature $T_{b b}$ at each of the calibration points using the SakumaHattori (SH) equation [9]. Here, $S_{b b}$ is the linearized signal after subtracting an e-plate 
measurement for each pixel, and $T_{b b}$ is the true blackbody values from Table 1 . While linearization converts a measured signal into a value of arbitrary units, the Sakuma-Hattori equation converts radiometric or apparent temperature to signal. Equation (7.1) shows that the function $F$ maps blackbody temperature $T_{b b}$ (in Kelvin) to camera signal from a blackbody measurement $S_{b b}$, and that this mapping is easily invertible. Equation (7.2) gives the SakumaHattori equations used in the linear regression and calibration in the forward form and (7.3) gives the inverse form, which gives the apparent temperature $T_{a p p}$. This is called apparent temperature because direct use of (7.3) to convert camera signal to temperature neglects the effects of emissivity or ambient temperature sources which are described in the measurement equation in Section 5.

$$
F: T_{b b} \leftrightarrow S_{b b}
$$

Forward

Sakuma-Hattori

Equation:

$$
F(T)=\frac{C}{\exp \left(\frac{c_{2}}{A T+B}\right)-1}
$$

Inverse Sakuma-

Hattori

Equation:

$$
F^{-1}(S)=T_{a p p}=\frac{c_{2}}{A \ln (C / S+1)}-\frac{B}{A}
$$

Here, $\mathrm{c}_{2}=14388 \mu \mathrm{m} \cdot \mathrm{K}$. Rather than fit the forward version of $F$ in (7.2), the non-linear regression fits the inverse function using calibration frame signal $S_{b b}$ as the independent variable and blackbody temperature $T_{b b}$ as the dependent variable. This also make a more proper fit when using a standard least squares algorithm since it is assumed the relative uncertainty of the blackbody true temperature $T_{b b}$ is less than the relative uncertainty of the calibration frame signal values. While $T_{b b}$ is developed from a traceable standard, $S_{b b}$ is subject to noise.

In fitting equation (7.3), the independent variable was weighted by $1 / S_{b b}$ to force the regression to favor the lower temperature values. It was later learned that the weights should be chosen based on the variance of the measurement errors, i.e., each weight value should be $w_{i}=1 / \sigma_{i}^{2}$, though these errors were not calculated a priori. Table 1 showed the frame noise is approximately $0.8 \%$ of the mean frame signal, making the chosen weights $1 / S_{b b} \propto 1 / \sigma$, instead of the preferred $1 / \sigma^{2}$. In [10], they chose weights based on the $0.05 \%$ relative signal uncertainties, though it wasn't stated if these were squared reciprocal, or just the reciprocal as done here.

By fitting the Sakuma-Hattori equation to calibration data, converting temperature to signal or vice versa simply requires evaluation of Equation (7). Though not done here, this curve-fitting may also make use of regression methods such as 'error-in-variables' or 'total least squares', or methods for determining confidence or prediction intervals for each pixel's calibration curve. Though these are more effective methods for evaluating calibration uncertainty, the method using standard error of the estimate (SEE) used in [1] is used here. However, as a single value 
for each pixel, the SEE does not give information on how the goodness of the regression varies with signal or temperature. A more arduous and future evaluation will determine and report prediction intervals with each pixel's curve-fit.

\subsubsection{Sakuma-Hattori Calibration Results}

A different 256 pixel x 320 pixel frame is stored for each SH coefficient and fit statistic similar to the linearization procedure outlined in Section 2.2. Table 3 gives the frame mean values for these arrays. Frame noise values are also given to demonstrate the variation in these values across the FPA. For example, the frame mean and frame noise of coefficient $A$ means $68.2 \%$ of all pixels resulted in an $A$ value of $1.9331 \pm 0.0606$. Or, $68.2 \%$ of all pixels resulted in an SEE of $0.0438 \pm 0.0149^{\circ} \mathrm{C}$.

Table 3: Sakuma-Hattori coefficients and fit statistics, and frame mean and frame noise of these values.

\begin{tabular}{ccc}
$\begin{array}{c}\text { SH Coefficient } \\
\text { or Fit Statistic }\end{array}$ & $\begin{array}{c}\text { Frame Mean } \\
\boldsymbol{\mu F}()\end{array}$ & $\begin{array}{c}\text { Frame Noise } \\
\boldsymbol{\sigma F}(0)\end{array}$ \\
\hline$A$ & 1.9331 & 0.0606 \\
$B$ & 25.9214 & 21.871 \\
$C$ & $7.8751 \mathrm{E} 06$ & $1.518 \mathrm{E} 06$ \\
$r^{2}$ & 0.9999515 & $3.94 \mathrm{E}-05$ \\
SEE $\left({ }^{\circ} \mathrm{C}\right)$ & 0.0438 & 0.0149
\end{tabular}

Though the inverse $\mathrm{SH}$ equation may result in temperatures below $300{ }^{\circ} \mathrm{C}$, levels below the lowest calibration point of $25 \Delta \mathrm{DL}\left(300^{\circ} \mathrm{C}\right)$ are not acknowledged. Though this example was given for one integration time $(550 \mu \mathrm{s})$, the coefficient A should scale with the integration time. However, this relationship becomes nonlinear as integration time decreases, so it is not recommended to extend a calibration done at one integration time to another solely by scaling by the integration time without cross-checking several calibration points.

The regression in Table 3 proved much better than that for the linearization in Table 2 based on the $r^{2}$ and relative SEE values. This is mostly due to the fact that the SH regression used linearized signal as the independent variable, and linearization had already acted as a nonuniformity correction and reduced the variance of all pixels in each frame. The average SEE of the SH curve-fit for all pixels is approximately $0.044^{\circ} \mathrm{C}$. Similar to the linearization, the SH curve-fitting acts as a per-pixel NUC. The example linearized calibration frames from Figure 5 and Figure 6 were converted to apparent temperature on a pixel-by-pixel basis using the inverse SH equation (Equation (7.3)) with results shown in Figure 7. 

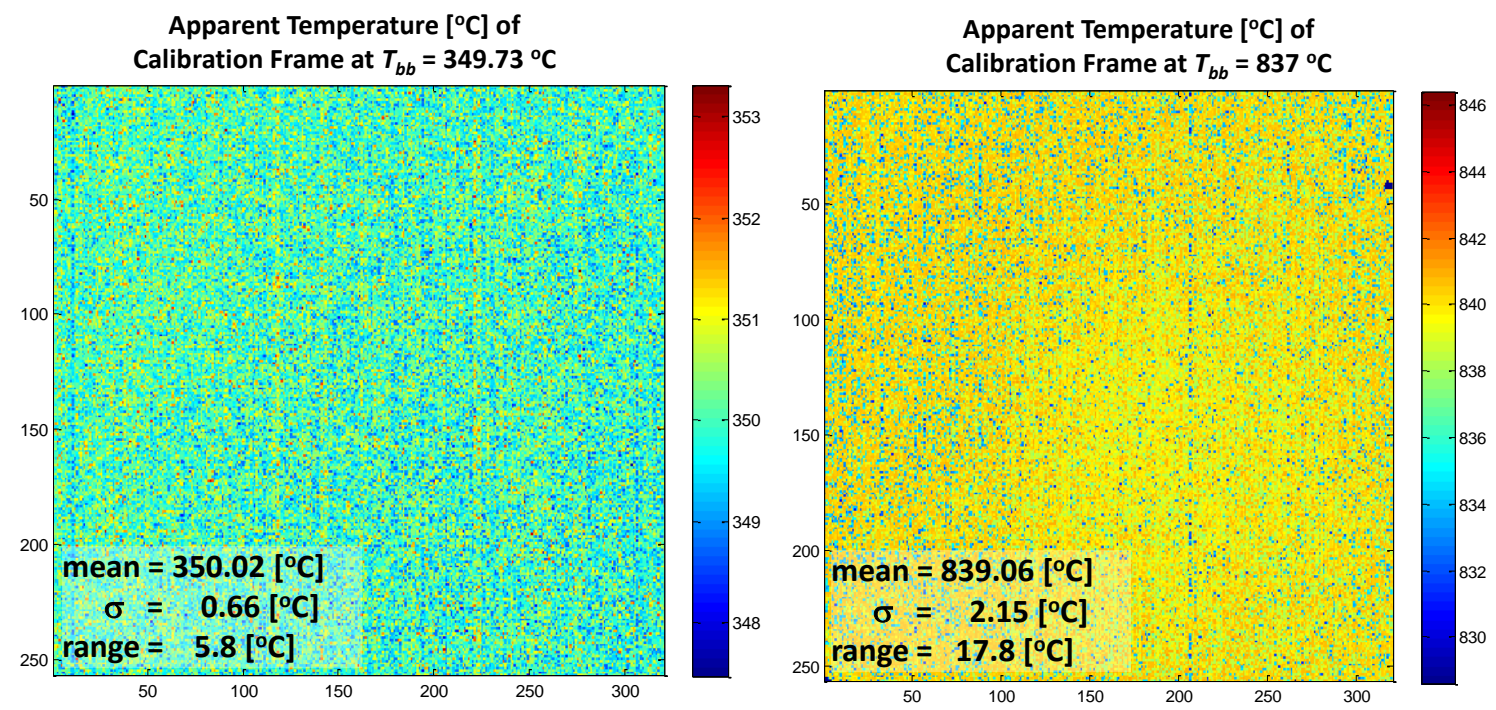

Figure 7: Comparison of a $T_{b b}=837^{\circ} \mathrm{C}$ calibration frame (left) and the linearized calibration (right) with frame mean, frame noise, and pixel value range.

For a perfect system, these apparent temperature frames should be uniform and equal to the true blackbody temperature $T_{b b}$. The calibration procedure error may be quantified by calculating the difference between the apparent temperature and $T_{b b}$. Figure 8 gives the frame mean of these values for each calibration point, and also shows the frame noise of each apparent temperature frame to give an idea of the relative non-uniformity of each frame.

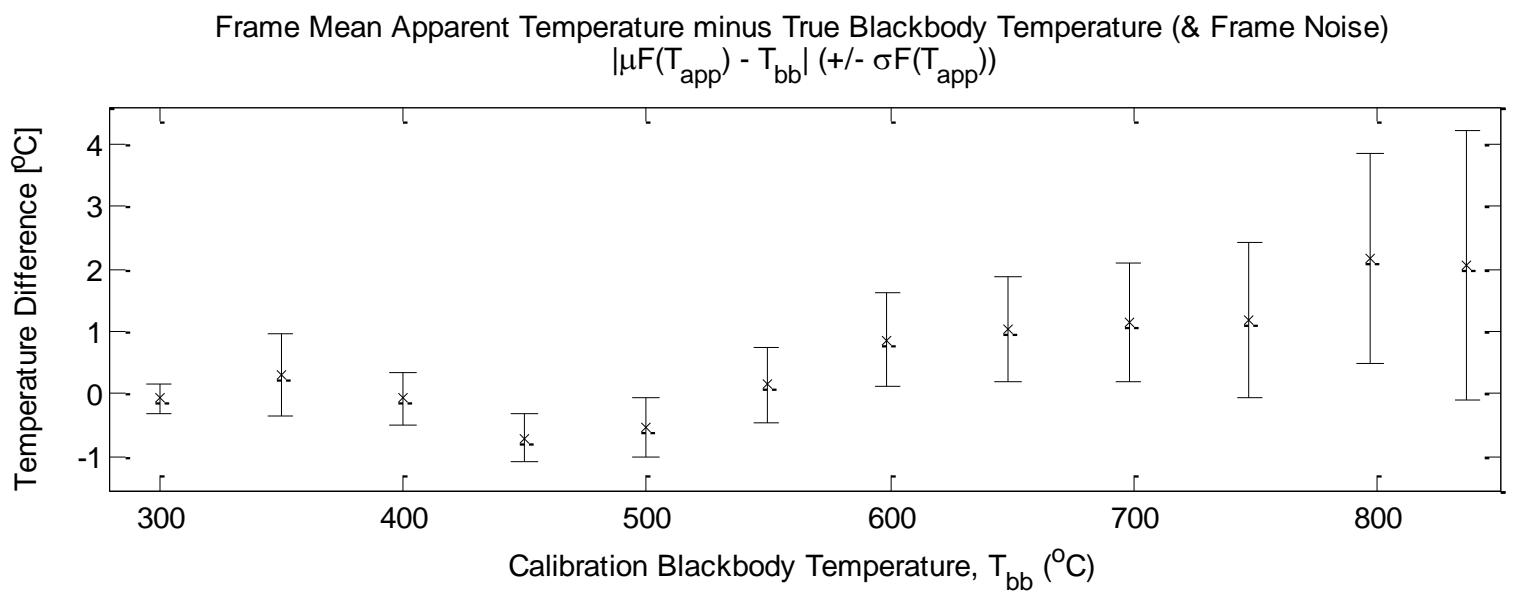

Figure 8: Temperature difference between calibration frames converted to apparent temperature (frame mean value) and the calibration blackbody temperature. Error bars indicate frame noise $(\sigma F)$ of each apparent temperature frame.

In Figure 8, the lower calibration points have smaller errors between apparent and blackbody temperatures. Recall that the Sakuma-Hattori curve-fits were weighted towards the lower temperatures, resulting in better signal to temperature conversion at these values. Also note that the increasing temperature frame noise with higher temperatures is a result of higher signal frame 
noise seen in Table 1. However, the relative magnitude of this noise is reduced when converting to temperature due to the nonlinearity of the inverse Sakuma-Hattori equation.

Saunders and White [11] gave formulae for estimating Sakuma-Hattori coefficients based on a detector's relative spectral responsivity. The results of these formulae are applied to the frame mean values from Table 3 to compare to values based on the camera responsivity given in Figure 3. These formulae for conversion are given in Appendix B.

\begin{tabular}{cccc} 
Parameter & $\begin{array}{c}\text { Based on SH } \\
\text { coefficients }\end{array}$ & $\begin{array}{c}\text { Based on } \\
\text { Responsivity }\end{array}$ & Units \\
\hline Central Wavelength $\lambda_{0}$ & 1.975 & 1.956 & $\mu \mathrm{m}$ \\
Variance, $\sigma^{2}$ & 0.012 & 0.023 & $\mu \mathrm{m}$ \\
Bandwidth, $\Delta \lambda$ & 0.373 & 0.525 & $\mu \mathrm{m}$ \\
Relative Bandwidth, $\sigma / \lambda_{0}$ & 0.055 & 0.077 &
\end{tabular}

While the central bandwidth $\lambda_{0}$ is well-determined by the calibration coefficients (to within 20 $\mathrm{nm}$ ), the bandwidth calculated based on the coefficients is approximately $30 \%$ narrower. For this camera, it would not be suggested to base calibration coefficients solely on the known responsivity, especially since the scaling ( $\mathrm{SH}$ coefficient ' $\mathrm{C}$ ' in Table 3) of each pixel varies across the FPA. However, when determining specifications of a potential camera system, these provide useful relationships between spectral bandwidth, system response, and their contributions to measurement uncertainty [1].

\section{POINT SPREAD FUNCTION MEASUREMENT}

Microscopic thermal imaging near the same scale as the detector wavelength (diffraction-limited scale) has the inherent problem of limited spatial resolution and blurry images. In thermography, where pixel temperature resolution as well as spatial resolution is important, blurring can cause significant error. High-temperature scenel areas may bias the apparent temperature of neighboring pixels higher, while in turn the high-temperature pixel is biased lower by the relatively cooler neighboring scenels. The scale of this effect depends on the local gradients of a particular measured scene and the intrinsic spatial resolution of the optical system defined by the point spread function (PSF). An 'ideal' image, or that without the optical blurring effects, may be extracted from a measured image by deconvolving a known or measured PSF from a collected image.

Point-spread function is defined as the resulting image from measuring an infinitesimally narrow point source, i.e., it is the impulse response of the optical system. Creating an impulse source to measure PSF is difficult in practice since reducing the width of a source to near zero will in turn reduce the intensity, and thus approach the noise floor of the system. Rather, measuring the edge spread function (ESF) is simpler, more common in practice, and recommended in ISO 12233 for 
characterizing optical system performance [12,13]. Edge spread function (ESF) is the image obtained when viewing the straight-line boundary between high and low intensity sources. It is related to the PSF through the line spread function (LSF), which is the image obtained when viewing an infinitesimally narrow and infinitely long line source. The ESF, LSF, and PSF are related as follows:

$$
\frac{d}{d x}[\operatorname{esf}(x)]=l s f(x)=\int_{-\infty}^{\infty} p s f(x, y) d y
$$

Another metric for characterizing optical system performance is the modulation transfer function (MTF, also called the optical transfer function). The MTF is the 2D Fourier transform of the $\mathrm{PSF}$, therefore defined in the spatial frequency domain. Frequency-domain characterization is not done here, though much information may be found $[8,12,14]$.

The ESF is measured by creating and imaging a knife edge (KE) scene, where a sharp, straight, opaque edge is placed at the focal plane between the camera and blackbody source. The KE image is used to create an $\operatorname{esf}(x)$ function by projecting all the KE image pixel values along the $\mathrm{KE}$ line into a super-sampled (i.e., data spacing is $<1$ pixel) digital ESF, then an appropriate esf $(x)$ function is fit to this data. This process is described further in the next section.

Once an ESF is determined, it is differentiated to determine the LSF. A further step is required in order to obtain the PSF from the LSF. PSFs may be approximated as rotationally symmetric for systems with rotationally symmetric optics and defined over a radius $(p s f(r))$ rather than Cartesian coordinates $(p s f(x, y))$. Marchand [15] gave multiple methods for converting an LSF to a rotationally symmetric PSF and vice versa, one of which noted that the LSF is the Abel transform of the radial cross-section of the corresponding rotationally symmetric PSF:

$$
\begin{array}{cc}
\begin{array}{l}
\text { Forward Abel } \\
\text { transform: }
\end{array} & l s f(x)=2 \int_{x}^{\infty} \frac{r \cdot p s f(r)}{\sqrt{r^{2}-x^{2}}} d r \\
\begin{array}{c}
\text { Inverse Abel } \\
\text { transform: }
\end{array} & p s f(r)=-\frac{1}{\pi} \int_{r}^{\infty} \frac{1}{\sqrt{x^{2}-r^{2}}}\left[\frac{d}{d x} l s f(x)\right] d x
\end{array}
$$

The rotationally-symmetric PSF function $p s f(r)$ may be calculated from the ESF measurement using Equations (8) and (9.2). The $p s f(r)$ function can then be digitally rotated about a center pixel to form the PSF image array.

Making use of certain properties of the Abel transform may allow more accurate evaluation of the ESF and transformation to the PSF. Abel transforms and their inverses (as well as the differential operator $d / d x$ ) are linear transformations [16]. Therefore, a linear combination of ESFs may be converted to a linear combination of LSFs and then PSFs. Tazannes and Mooney [14] mentioned the possibility of using a linear combination of functions to fit an ESF, however 
they did not detail how this was a mathematically sound approach. By fitting a linear combination of ESF functions, the number of fit parameters may be increased until a satisfactory fit is made. In addition, linear combinations of different potential ESF functions may be used. This property of the PSF is exploited here.

\section{Procedure for calculating the PSF:}

1.) Create a knife edge scene in front of the blackbody source and collect a KE image in DLs. Collect a corresponding e-plate measurement.

2.) Subtract the e-plate measurement from the $\mathrm{KE}$ image, then linearize the $\mathrm{KE}$ image to convert from $\triangle \mathrm{DLs}$ to a.u.

3.) Project the pixel values along the knife edge to form the supersampled ESF

4.) Fit various linear combinations of potential ESF functions to the supersampled ESF. Select the best fit as the esf(x).

5.) Convert the esf(x) into a $p s f(r)$ using the inverse Abel transform.

6.) Digitally rotate and resample the $2 \mathrm{D} p s f(r)$ to form a PSF image array.

Once a PSF is determined, an image may be deblurred by deconvolving the PSF array from the measured image. Note that all of this is done with linearized signal (e.g., proportional to radiant intensity) before converting to temperature.

\subsection{Measuring the ESF}

\subsubsection{Measurement and Projection of the KE image}

The ESF as defined is perpendicular to the KE line. However, if the KE is aligned vertically or horizontally with the FPA, the location of the KE line will lie in the middle of a row of pixels, therefore cannot be determined to better than one pixel width. Tilting the KE diagonally across the scene is preferred. Multiple methods may be used to locate the edge in the image, mostly relying on some method of differentiating the image along some direction, and locating the maximum of this gradient, and fitting a line to the maxima locations. An example is given in Figure 9, which was made by placing a razor blade at the focal plane in front of a $700{ }^{\circ} \mathrm{C}$ blackbody source. The result of fitting a line to these points is the slope intercept form of the KE line, i.e., $\mathrm{j}=\mathrm{mi}+\mathrm{b}$, where $\mathrm{j}$ and $\mathrm{i}$ are pixel indices, and $\mathrm{m}$ and $\mathrm{b}$ are slope and intercept.

According to ISO 12233, a super-sampled ESF is generated from the KE image by projecting pixel values along the KE line onto a unified axis. This axis is defined by distance between a pixel and the KE line (therefore, making 0 the center of the knife edge and ESF). However, ISO 12233 also states that the KE line should only be tilted $5^{\circ}$ from the vertical and the horizontal distance from pixel to $\mathrm{KE}$ line is used as the projection axis. Assuming a $5^{\circ}$ tilt is equivalent to a vertical knife edge is computationally easier, though here we use the real normal (or minimum) distance to the KE line, as shown in Figure 9, and calculated in Equation (10). 


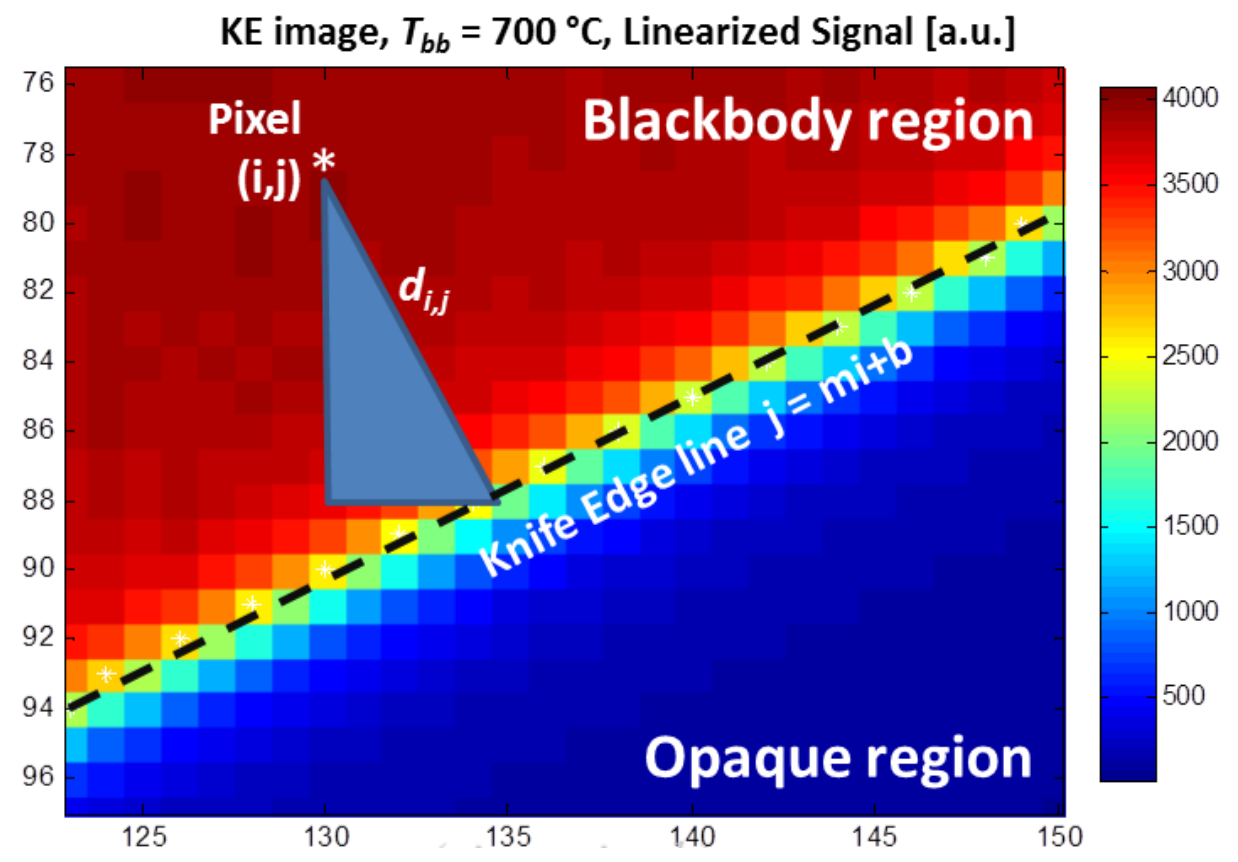

Figure 9: Example knife edge image showing the KE line fit to image gradient maxima (denoted by a small white '+' in the middle of a pixel). Calculation for determining each pixel's normal distance the KE line is also shown.

$$
d_{i, j}=\frac{j-m i-b}{\sqrt{m^{2}+1}}
$$

Plotting pixel values (linearized signal, [a.u.]) vs. this distance results in the super-sampled ESF, shown in Figure 10 with three regions $(a, b$, and $c)$ highlighted in subfigures. This was done for three blackbody temperature values. These values are normalized, as the vertical scale of the ESF is not important, and later PSF values are normalized as well. 

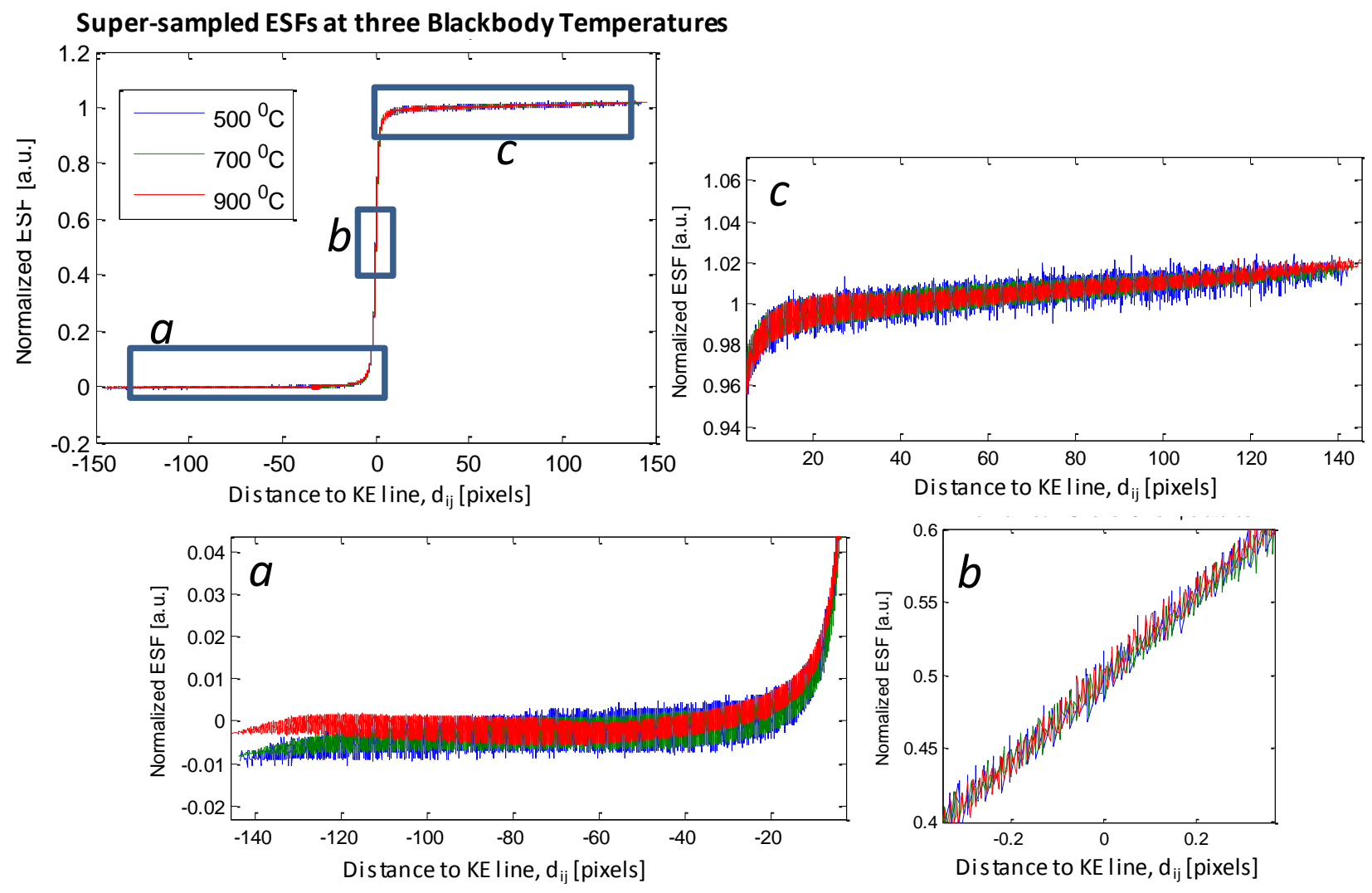

Figure 10: Three super-sampled ESFs (normalized) at different blackbody temperatures.

The $900{ }^{\circ} \mathrm{C}$ supersampled ESF appeared similar to the others at the higher end, representing the optically clear region of the knife edge image, but different in the lower, opaque region. It is believed that the razor blade may have heated up while in front of the blackbody, thus having radiant emission levels higher than ambient condition. ${ }^{1}$ The $900{ }^{\circ} \mathrm{C}$ ESF is considered erroneous and not analyzed further. Due to the similarity between the $500^{\circ} \mathrm{C}$ and $700{ }^{\circ} \mathrm{C} \mathrm{ESFs}$, only the $700{ }^{\circ} \mathrm{C} \mathrm{ESF}$ is further analyzed.

At this point, ISO 12233 recommends the supersampled ESF be re-sampled into 'bins' where all points lying within $1 / 4$ pixel increments are averaged together in order to reduce noise by averaging, but still provide $4 \mathrm{x}$ supersampled data to reduce aliasing. However, this document departs from ISO 12233 at this point, and fits a smooth, continuous curve to the super-sampled ESF data. One of the purposes of the ISO 12233 is to develop the MTF in order to characterize and compare camera systems. However, here we intend to develop a PSF to use as a deconvolution kernel to de-blur thermographic images.

\footnotetext{
${ }^{1}$ It should be noted that the lower (opaque) region of the ESF had camera signal values very near the lower boundary of the calibration ( $0 \Delta \mathrm{DL}$ to $20 \Delta \mathrm{DL}$ ), while the higher (transparent) region varies in magnitude with the blackbody temperature. A more appropriate method may be to use a semi-transparent knife edge so that lower signal levels are in the more linear range and scale with the higher region and blackbody temperature.
} 


\section{Curve-fitting the ESF:}

Multiple authors have noted that common functional forms of the ESF resemble cumulative distribution functions, and claim the LSF or PSF are the associated probability distributions. Smith [17] attempted multiple single ESF functions (i.e., not a linear combination) that were fit to a measured ESF. These included error function (which generates a Gaussian distribution PSF) and arctangent (which generates a Cauchy distribution PSF). Tazannes and Mooney [14] fit a linear combination of Fermi distributions to a measured ESF.

Various combinations of error function and arctangent function forms were fit to the supersampled ESF data. Expressions for $l s f(x)$ were determined by simple differentiation, and conversion from $l s f(x)$ to $p s f(r)$ through inverse Abel transform utilized tables of calculated transforms $[16,18]$. These functions are shown in Table 4. For single-function fitting of ESFs, any scaling coefficient is unimportant since the ESF and generated PSF may be eventually normalized. However, with linear combinations of ESF function, the proper Abel transform is important to maintain the relative scaling effect of each coefficient in the linear combination.

Table 4: ESF, LSF, and PSF relationships based on the assumption of Gaussian or Cauchy distribution shaped PSFs.

Gaussian PSF:

$$
\begin{aligned}
& \operatorname{esf}(x)=\frac{1}{2}+\sum_{i=1}^{N} a_{i} \cdot \operatorname{erf}\left(\frac{x}{b_{i}}\right) \\
& \operatorname{lsf}(x)=\frac{2}{\sqrt{\pi}} \sum_{i=1}^{N} \frac{a_{i}}{b_{i}} \cdot \exp \left(\frac{-x^{2}}{b_{i}^{2}}\right) \\
& \operatorname{psf}(r)=\frac{2}{\pi} \sum_{i=1}^{N} \frac{a_{i}}{b_{i}^{2}} \cdot \exp \left(\frac{-r^{2}}{b_{i}^{2}}\right)
\end{aligned}
$$

\section{Cauchy PSF:}

Five linear combinations were attempted and are given in Table 5. These included from one to two Cauchy functions and one to three Gaussian functions. Mixed combinations of Gaussian and Cauchy PSFs were not attempted. Linear combinations of Gaussians provided superior fits. After the 3-Gauss function resulted in an RMS error of less than $0.2 \%$ full scale of the ESF, further attempts at fitting more complex ESF combinations were ceased. The multiple curve fits to the supersampled $700{ }^{\circ} \mathrm{C} \mathrm{ESF}$ are shown in Figure 11, with lower, central, and upper region expanded for clarity. Resulting $p s f(r)$ functions, normalized to the area under the curve, are plotted in Figure 12. 


\section{ESF Fit Functions}
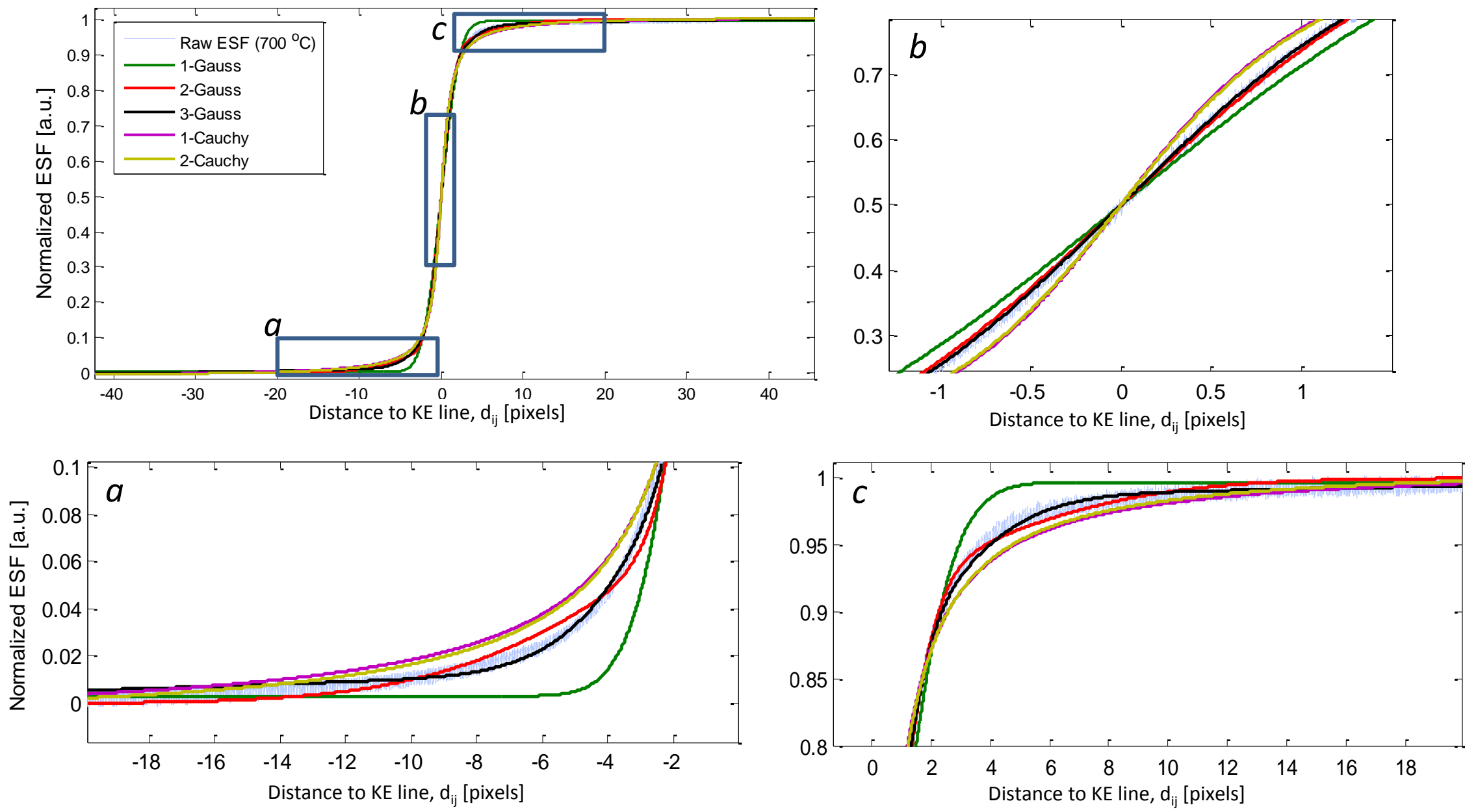

Figure 11: Curve fits to the $700{ }^{\circ} \mathrm{C} \mathrm{ESF}$ using various linear combinations of fit functions. 
Table 5: Fit results for the five tested ESF functions.

\begin{tabular}{|c|c|c|c|c|c|c|}
\hline Name & ESF Function (fit) & PSF functon & Coefficients & & $r^{2}$ & RMSE \\
\hline \multirow[t]{2}{*}{ 1-Gauss } & a1*erf(x./b1)+0.5 & $2^{*} a 1 / p i / b 1^{\wedge} 2^{*} \exp \left(-r^{\wedge} 2 / b^{\wedge} 2\right)$ & a1 & 0.496 & 0.99949 & 0.0050 \\
\hline & & & b1 & 2.475 & & \\
\hline \multirow[t]{4}{*}{ 2-Gauss } & a1*erf(x./b1) + & $2^{*} \mathrm{a} 1 / \mathrm{pi} / \mathrm{b} 1^{\wedge} 2^{*} \exp \left(-\mathrm{r} .^{\wedge} 2 / \mathrm{b} 1^{\wedge} 2\right)+$ & a1 & 0.088 & 0.99989 & 0.0023 \\
\hline & a2*erf(x./b2)+0.5 & $2^{*} \mathrm{a} 2 / \mathrm{pi} / \mathrm{b} 2^{\wedge} 2^{*} \exp \left(-\mathrm{r} .{ }^{\wedge} 2 / \mathrm{b} 2^{\wedge} 2\right)$ & a2 & 0.412 & & \\
\hline & & & b1 & 8.959 & & \\
\hline & & & b2 & 1.871 & & \\
\hline \multirow[t]{6}{*}{ 3-Gauss } & $a 1^{*} \operatorname{erf}(x . / b 1)+$ & $2^{*} \mathrm{a} 1 / \mathrm{pi} / \mathrm{b} 1^{\wedge} 2^{*} \exp \left(-\mathrm{r}^{\wedge} 2 / \mathrm{b} 1^{\wedge} 2\right)+$ & a1 & 0.327 & 0.99994 & 0.0017 \\
\hline & a2*erf(x./b2) + & $2^{*} \mathrm{a} 2 / \mathrm{pi} / \mathrm{b} 2^{\wedge} 2^{*} \exp \left(-\mathrm{r}^{\wedge} 2 / \mathrm{b} 2^{\wedge} 2\right)+$ & a2 & 0.019 & & \\
\hline & a3*erf(x./b3)+0.5 & 2*a3/pi/b3^2*exp(-r.^2/b3^2) & a3 & 0.158 & & \\
\hline & & & b1 & 1.577 & & \\
\hline & & & b2 & 43.095 & & \\
\hline & & & b3 & 4.692 & & \\
\hline \multirow[t]{2}{*}{ 1-Cauchy } & $\mathrm{a} 1 / \mathrm{b} 1 * \operatorname{atan}(\mathrm{x} / \mathrm{b} 1)+0.5$ & $a 1 / 2^{*}\left(b 1^{\wedge} 2+r .^{\wedge} 2\right) .^{\wedge}-1.5$ & a1 & 0.295 & 0.99976 & 0.0035 \\
\hline & & & b1 & 0.908 & & \\
\hline \multirow[t]{4}{*}{ 2-Cauchy } & $\mathrm{a} 1 / \mathrm{b} 1 * a \tan (\mathrm{x} / \mathrm{b} 1)+$ & $a 1 / 2^{*}\left(b 1^{\wedge} 2+r .^{\wedge} 2\right) \cdot{ }^{\wedge}-1.5+$ & a1 & 0.307 & 0.99980 & 0.0032 \\
\hline & $a 2 / b 2 * \operatorname{atan}(x / b 2)+0.5$ & $a 2 / 2^{*}\left(b 2^{\wedge} 2+r .^{\wedge} 2\right) .^{\wedge}-1.5$ & a2 & -1337.412 & & \\
\hline & & & b1 & 0.936 & & \\
\hline & & & b2 & 3929.574 & & \\
\hline
\end{tabular}

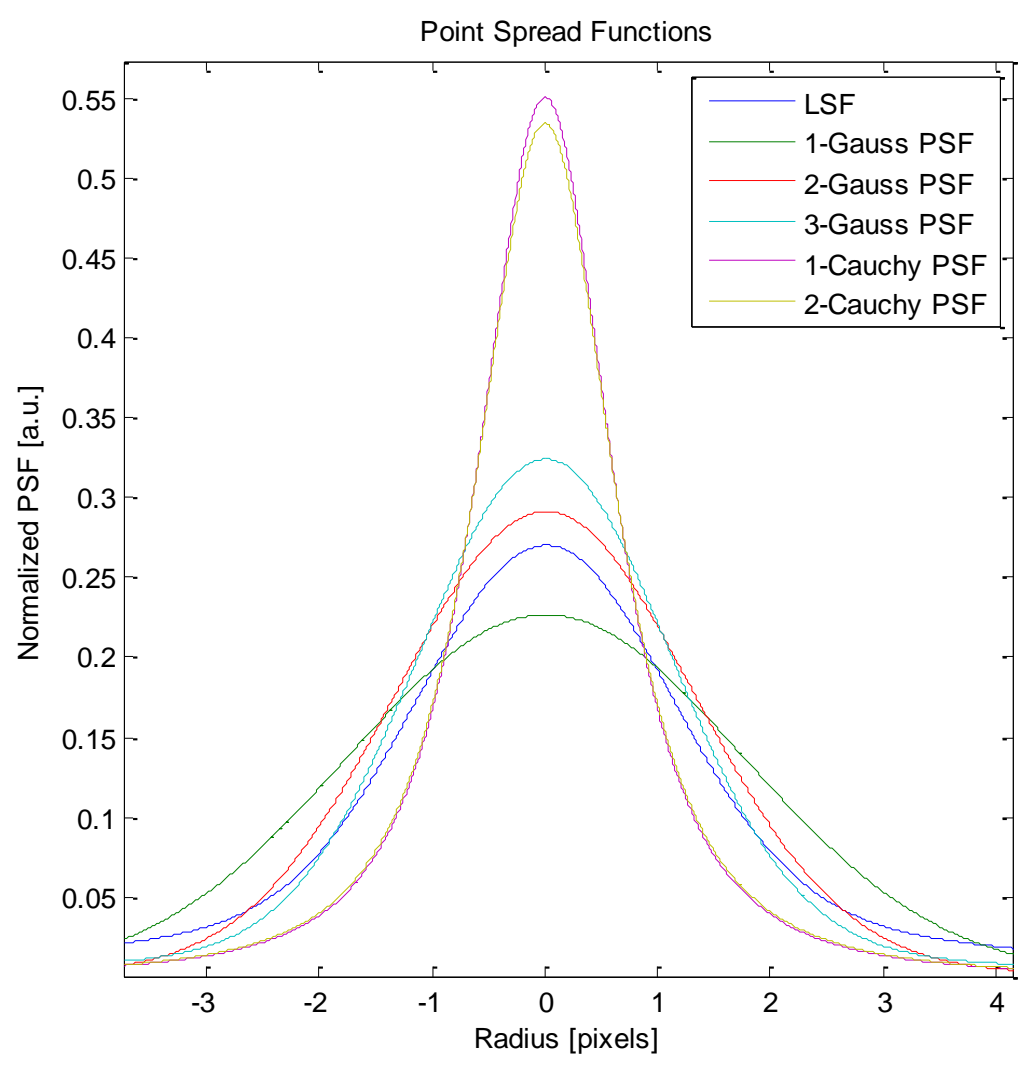

Figure 12: Resulting PSFs, normalized based on the 2D area under the curve 


\subsection{Formation of the 3D PSF}

All calculations here, including integration, are done numerically. The smooth, algebraic function $p s f(r)$ given in Table 5 is oversampled to 10/pixel, and spline interpolation used to determine intermediary values. To 'digitally rotate' and re-sample $p s f(r)$ into an PSF image array, the radius value is converted back into Cartesian coordinates and integrated over each pixel of coordinates $(\mathrm{i}, \mathrm{j})$ :

$$
P S F_{i, j}=\int_{j-0.5}^{j+0.5} \int_{i-0.5}^{i+0.5} p s f\left(\sqrt{x^{2}+y^{2}}\right) d x d y
$$

After generating the PSF image array, it is normalized such that the volume under the PSF is unity. Figure 13 (left) demonstrates how Equation (12) develops the rotated and digitized PSF array. It shows the central 5 pixel $x 5$ pixel region, though the PSF array is defined over a 41 pixel $x 41$ pixel array. The majority of the PSF occurs over the central 7 pixel diameter, with values quickly tapering off outside this region as seen in the logarithmic scaled plot in Figure 13 (right). Despite their low magnitude, these exterior pixels are important due to the cumulative nature of 2D convolution and deconvolution. The 41 pixel $x 41$ pixel size of the PSF was chosen arbitrarily, but ensured that it encompassed all the PSF data to within four orders of magnitude. Also visible is the transition between the different contributing Gaussian functions that compose the linear combination PSF detailed in Table 5.
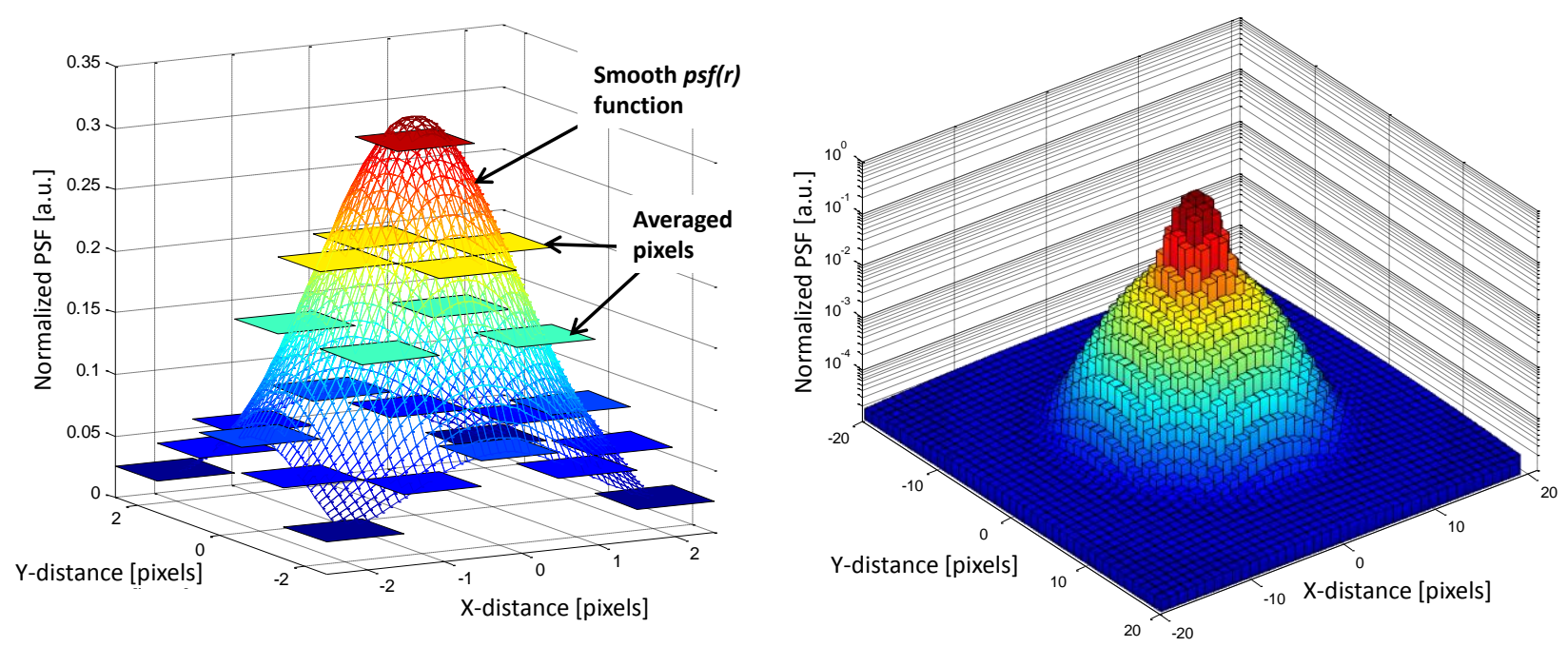

Figure 13: Left: Demonstration of how central region of the smooth $p s f(r)$ function is averaged over each pixel to develop the PSF image array. Right: The full PSF image array plotted on a logarithmic scale for detail.

\subsection{PSF Deconvolution and Results}

To test the PSF measurement, a calibration image was taken. This is made by placing a glass 1951 US Air Force standard resolution chart (or Air Force target, AFT) in front of the calibration 
blackbody. Because it was a glass target, the signal is attenuated, so signal values do not correspond to the blackbody temperature. Nonetheless, the target is still useful for determining the attenuation of small targets (triplet bars of multiple sizes on the AFT) due to blurring from the PSF. Table 6 gives the bar sizes for each element in each group of the AFT.

Table 6: Bar width for group and element numbers in the 1951 AFT used to test the PSF

\begin{tabular}{ccccc}
\multicolumn{5}{c}{ Bar width $(\boldsymbol{\mu m})$} \\
Element & Group 4 & Group 5 & Group 6 & Group 7 \\
\hline 1 & 31.3 & 15.6 & 7.8 & 3.9 \\
2 & 27.8 & 13.9 & 7.0 & 3.5 \\
3 & 24.8 & 12.4 & 6.2 & 3.1 \\
4 & 22.1 & 11.0 & 5.5 & 2.8 \\
5 & 19.7 & 9.8 & 4.9 & 2.5 \\
6 & 17.5 & 8.8 & 4.4 & 2.2 \\
\hline
\end{tabular}

Before the linearized signal AFT image is deconvolved with the PSF, it is symmetrically mirrored on each edge by the width of the PSF window (41 pixels) to reduce any edge effects of the deconvolution algorithm. The AFT image is then deblurred using a blind deconvolution (maximum likelihood algorithm) with the 3-Gauss PSF array as an initial PSF. Figure 14 shows the results of the AFT image with and without PSF correction, converted to apparent temperature. Two sub-regions are expanded to show the de-blurring effect of the PSF deconvolution. 

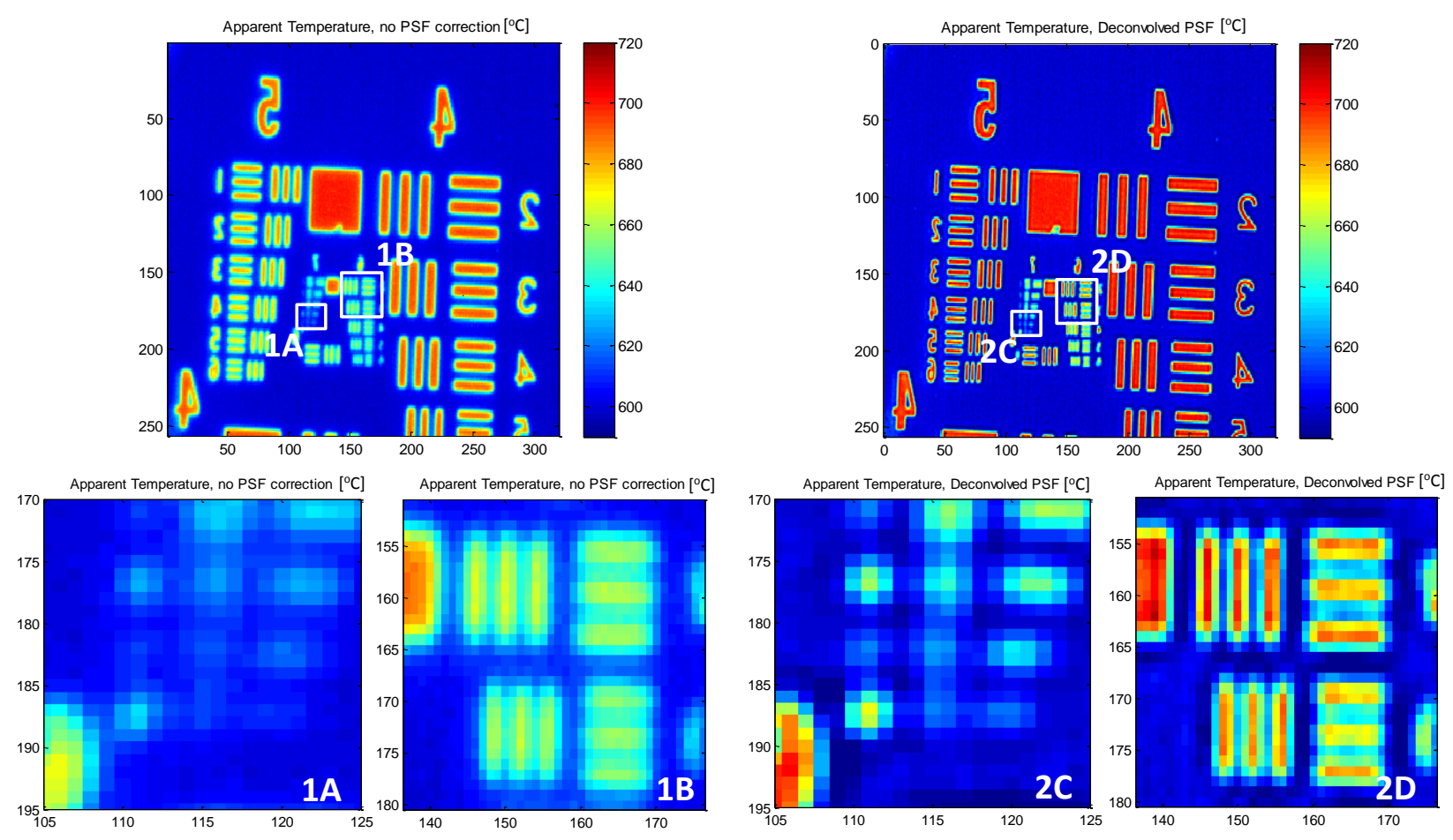

Figure 14: Example AFT image converted to apparent temperature without PSF deconvolution (left top image) and with PSF deconvolution (right top image), showing the deblurring effect on small features (bottom row).

Three traces are made of the AFT image to better observe the effect on temperature values vs. bar width. Figure 15 gives the results. The bar elements in the subfigures 1B and 2D in Figure 14 are group 6, element 2 and 3, with $7.0 \mu \mathrm{m}$ and $6.2 \mu \mathrm{m}$ bar spacing, respectively. These are also shown in Trace \#3 in Figure 15. 


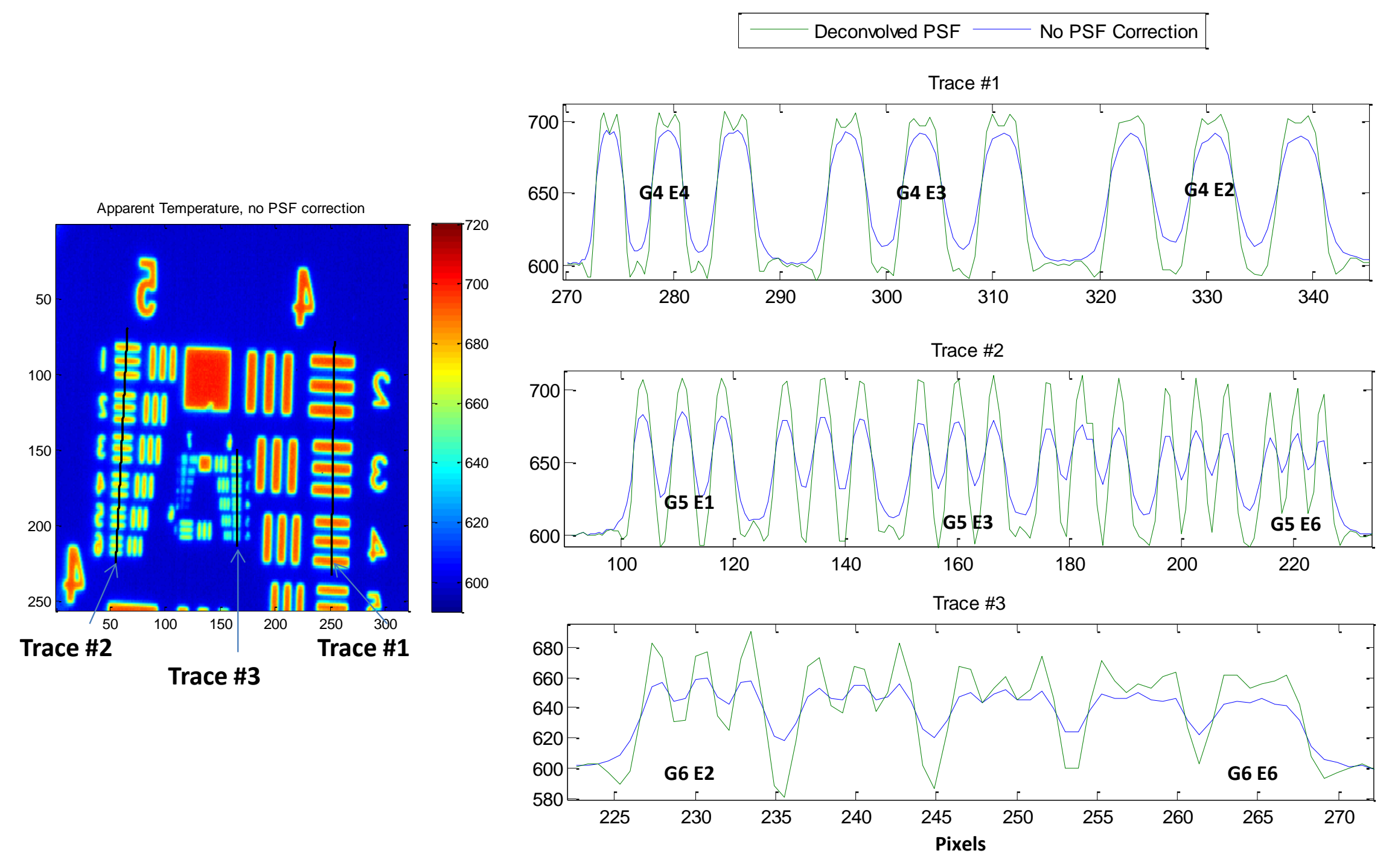

Figure 15: Example traces of the AFT image (left) with and without PSF correction. Group and element numbers are given in each trace plot for select bar elements. 
Figure 14 and Figure 15 show that the temperature attenuation on the smaller bar groups caused by the PSF may be corrected. The limit may be seen at approximately group 5 element 3 (bar widths of $12.4 \mu \mathrm{m}$ ). For bars wider than this, the full $100{ }^{\circ} \mathrm{C}$ range of temperature is reproduced without attenuation. Narrower than this width, the bars are attenuated even with PSF correction. However, for bar elements as narrow as $4.4 \mu \mathrm{m}$ (group 6 element 6), PSF deconvolution decreases the attenuation effect.

In [1], it was found that for an example scene and PSF and measuring the maximum pixel temperature value, PSF correction provided an error of only $-8{ }^{\circ} \mathrm{C}$ from the true maximum temperature of $973{ }^{\circ} \mathrm{C}$, whereas without PSF correction, the error was $-105^{\circ} \mathrm{C}$. The component of uncertainty due to error in the 'measured' PSF, as defined in [1], was $10.5^{\circ} \mathrm{C}$. In [1], the errors were based on a simulated image and a simulated PSF and PSF uncertainty. However, accomplishing the inverse problem of evaluating error from a measured image and measured PSF is still under investigation. It was shown, however, that PSF correction is necessary to reduce the biasing effect of optical blur on measurement error, and accurate measurement of the PSF is key to reducing the uncertainty due to this correction.

Motion blur was also investigated in [1]. This occurs when objects in the measured scene are moving or vibrating at a frequency such that blurring or persistence of motion occurs [2]. This blur increases with the velocity of the object, and decreases with the camera integration time. Though not mentioned here, it may be necessary to either compensate for motion blur by measuring its effect on the camera system, measuring the motion or vibration of the measured object and devising an appropriate Type-A uncertainty, or providing a Type-B uncertainty estimate [19].

\section{EMISSIVITY MEASUREMENT}

At this point, images may be collected, PSF-corrected, and converted to apparent temperature using the linearization and calibration scheme in Section 2. However, to convert to true temperature, the signal values need to be corrected for emissivity. It was noted in [1] that uncertainty in emissivity accounts for the largest source of measurement uncertainty. Therefore, more effort is taken here to provide better emissivity measurements. Madding [20] mentioned several methods for measuring emissivity; using a reference emitter, a reference temperature, measuring the target at two temperatures, or measuring reflectivity. In the authors' previous work, only the reference temperature method was used by measuring the target temperatures with a thermocouple. Here, each method is used (except measuring reflectivity), although not always simultaneously. This depends if a reference emitter is available on the particular sample being measured. The reference emitter method is accomplished by electro-discharge machining (EDM) a small hole in the face of the tool shown in Figure 16. When the insert is heated, this hole acts as a micro-blackbody $(\mathrm{mBB})$, with a reference emissivity value very near unity. 
The EDM process achieved mBB diameters of approximately $0.3 \mathrm{~mm}$, with depths greater than $3.5 \mathrm{~mm}$ measured by sticking a fresh, $0.2 \mathrm{~mm}$ EDM electrode into the finished $\mathrm{mBB}$ and measuring the displacement from the surface in a shadowgraph. It is well known that cylindrical cavities form blackbodies with effective emissivities greater than 0.990 if the depth to diameter ratio is greater than 10 and internal wall emissivity is greater than 0.5 [21].

Several steps are taken to prepare and heat the tool during emissivity measurement. After the $\mathrm{mBB}$ is made, the tools are ultrasonically cleaned in an acetone bath. From this point forward, any contact with the tool is made with cotton or nitrile gloves to prevent skin oil from contaminating the surface. The tool is wrapped tightly with copper foil, shown in Figure 16, with a type-K thermocouple held in close contact with the approximate center of the insert face. This is then wrapped in 18 gauge nichrome wire as the heat source. The copper foil better distributes heat from the nichrome around the insert. The nichrome-wrapped insert is held between two copper jaws in a vise (called the 'thermal vise'), which are connected to a pulsed reflow soldering power controller $(4.0 \mathrm{KV} \cdot \mathrm{A}$ power). This controller allows timed, feed-back controlled (via thermocouple) heating up to $1000{ }^{\circ} \mathrm{C}$ by passing controlled current from the vise jaws through the nichrome wire causing it to heat.

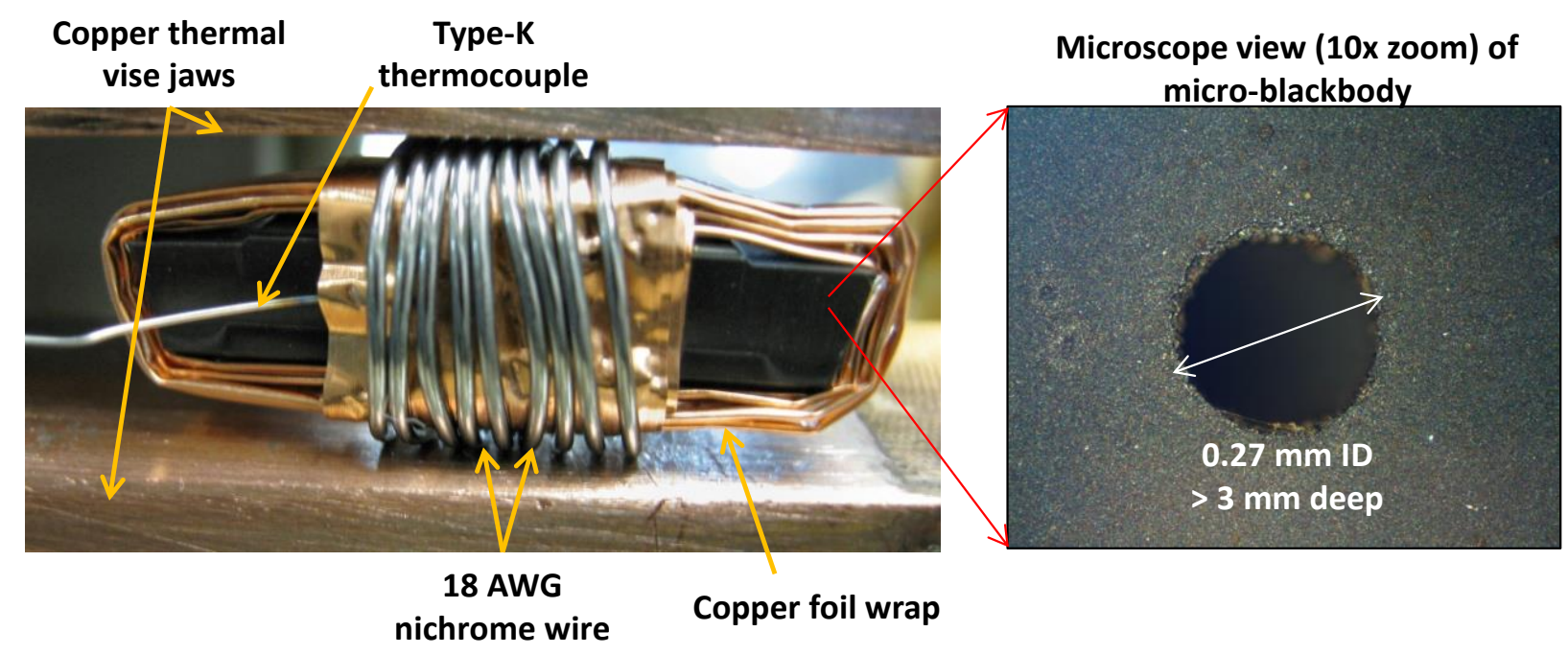

Figure 16: Example of a tungsten carbide tool in the thermal vise heating apparatus during emissivity measurement. Temperature and reference radiance are measured via thermocouple and/or micro-blackbody

While in the thermal vise, the tool is heated in cycles lasting $200 \mathrm{~s}$ each. These cycles increase in temperature from $100{ }^{\circ} \mathrm{C}$ to $550{ }^{\circ} \mathrm{C}$ for a total of 25 cycles. While developing this method, it was found that discoloration through oxidation, as well as emissivity values, ceased to change after approximately 20 cycles. Images of the $\mathrm{mBB}$ are taken at nominally $550{ }^{\circ} \mathrm{C}$ and another cycle at $450{ }^{\circ} \mathrm{C}$. If a $\mathrm{mBB}$ is not on a sample, then the bare face of the insert is measured at nominally $550{ }^{\circ} \mathrm{C}$ and $450{ }^{\circ} \mathrm{C}$. After the emissivity images are taken, the raw signal image is 
linearized using the methods from Section 2, and PSF corrected using the PSF measured in Section 3.

Figure 17 gives an example of two mBB images taken at two thermocouple temperatures $\left(T_{T C}\right)$. To get camera signal values representing the $\mathrm{mBB}$ radiance, a region of interest (ROI) is selected within the $\mathrm{mBB}$, and mean signal pixel value, $S_{m B B}$, is calculated (as well as standard deviation $\sigma)$. An ROI outside the $\mathrm{mBB}$ is also made, with mean signal pixel value calculated for the tool region $\left(S_{\text {tool }}\right)$. These ROIs were separated to avoid the transition boundary between the tool region and micro-blackbody region, and a noticeable ring around the micro-blackbody. The source of the ring was undetermined, though it is hypothesized that this was residue from the EDM process.
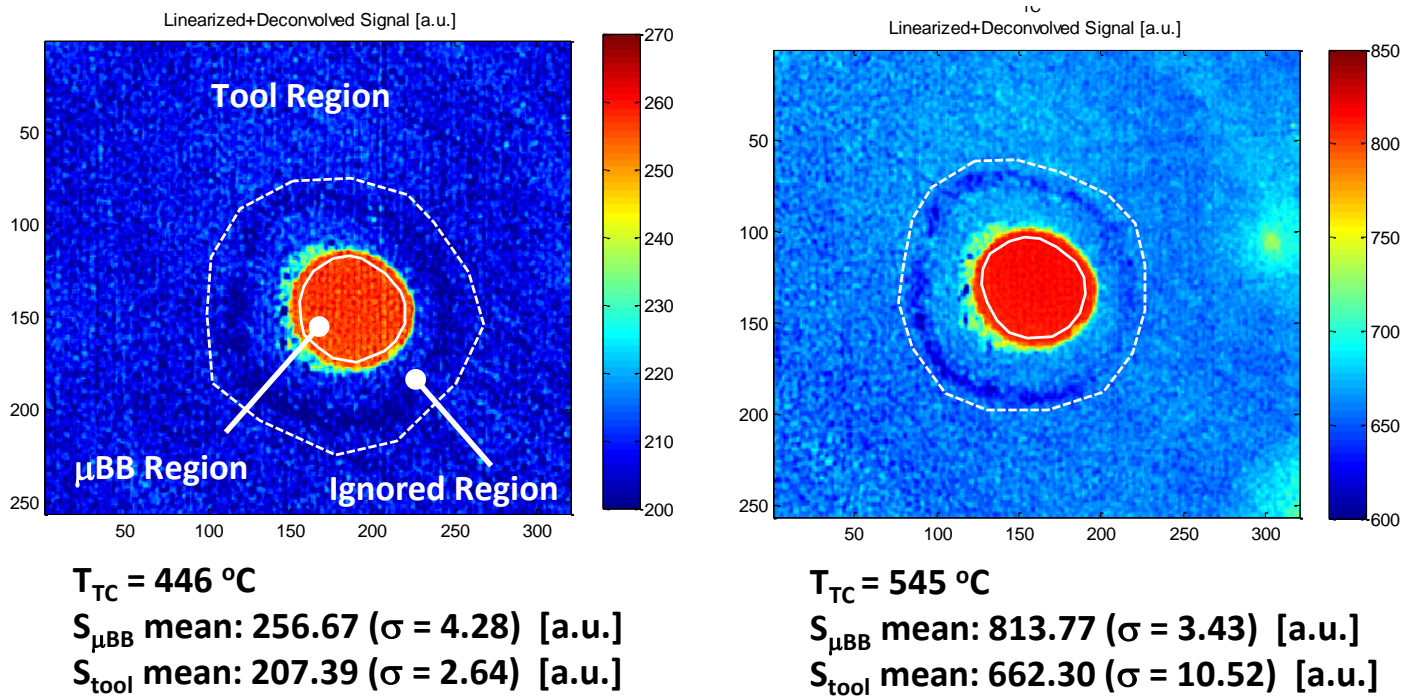

Figure 17: Example micro-blackbody signal images (in [a.u.]) and user-selected regions of interest (ROIs) used to measure mBB signal and signal on the heated tool face.

Right away, it was noticed that the $\mathrm{mBB}$ and thermocouple gave differing temperature values for each insert measured. For example, in Figure 17, the mean signal measured in the mBB region $\left(\mathrm{S}_{\mathrm{mBB}}\right)$ converted to apparent temperature using the inverse calibration discussed in Section 2.3 give mBB temperatures of $435{ }^{\circ} \mathrm{C}$ and $525^{\circ} \mathrm{C}$; lower than the thermocouple readings of $446{ }^{\circ} \mathrm{C}$ and $545^{\circ} \mathrm{C}$, respectively. Two things may cause this; 1 ) the thermocouple is in fact at a higher temperature than the micro-blackbody or 2) the micro-blackbody has an emissivity less than the expected $>0.990$ value $^{2}$.

Reason 1 is taken as true. The thermocouple lies under highly conductive copper foil which brings the thermocouple in closer thermal proximity to the nichrome heat source. The mBB

\footnotetext{
${ }^{2}$ If the thermocouple temperatures are regarded as true, and the $\mathrm{mBB}$ is the same temperature, then this would result in a mBB emissivity of approximately 0.85 . From previous work at NIST [22], tool emissivity was measured near 0.85, whereas the $\mathrm{mBB}$ in Figure 17 is visibly emitting more than the surrounding tool face. This further gives reasoning for $T_{T C}$ being an inaccurate measure of the tool face temperature.
} 
occurs on an exposed face which is subject to radiative and convective cooling, while the thermocouple lies under the copper foil. In addition, the high depth to diameter ratio of the $\mathrm{mBB}$ discussed previously ensures relatively high effective emissivity. Due to the potential difference between thermocouple and tool face temperatures, previously unconsidered in [1], it is noted that thermocouples should be placed very near the $\mathrm{mBB}$ on the exposed insert face; preferably fixed via resistance spot welding. Though this discrepancy is known here, methods for calculating emissivity based on the mBB, thermocouple, or both are given in the next section.

\subsection{Calculating Emissivity}

Values used to calculate emissivity are the micro-blackbody signal $\left(S_{m B B}\right)$, micro-blackbody signal converted to temperature $\left(T_{m B B}\right)$, or the thermocouple temperature $\left(T_{T C}\right)$. Multiple methods of calculating emissivity based on these measurements require certain assumptions of varying feasibility. Assuming negligible ambient reflection, measured signal from the heated tool face or the micro-blackbody is related to their respective temperatures, $T_{\text {tool }}$ and $T_{m B B}$ :

$$
\begin{gathered}
S_{\text {tool }}=\varepsilon_{\text {tool }} F\left(T_{\text {tool }}\right) \\
S_{m B B}=\varepsilon_{m B B} F\left(T_{m B B}\right)
\end{gathered}
$$

If it is assumed that the tool face temperature is well represented by the thermocouple temperature, then $T_{\text {tool }}=T_{T C}$, and emissivity is:

$$
\varepsilon_{\text {tool }}=\frac{S_{\text {tool }}}{F\left(T_{T C}\right)}
$$

If a micro-blackbody is available, then one may assume that the tool surface and internal temperature are uniform and equal, or $T_{\text {tool }}=T_{m B B}$. Then combining Equations (13.1) and (13.2) yields the following, noted in [20] as the 'reference emitter' technique:

$$
\varepsilon_{\text {tool }}=\varepsilon_{m B B} \frac{S_{\text {tool }}}{S_{m B B}}
$$

The assumption can be made that $\varepsilon_{\mu B B}=1$ in Equation (15). If it is assumed $\epsilon_{\mathrm{mBB}} \neq 1, \epsilon_{\mathrm{mBB}}$ can be calculated from (13.2). However, $T_{m B B}$ is unknown. If you assume $T_{\mu B B}=T_{T C}$, then (13.2) gives the following:

$$
\varepsilon_{m B B}=\frac{S_{m B B}}{F\left(T_{T C}\right)}
$$

Inserting Equation (16) into (15) yields (14), thereby requiring the same assumptions. If measurements are taken at more than one temperature, it may be assumed that the emissivity is the same at the two temperature $(\Delta \epsilon=0)$. The two temperature technique noted in [20] may be calculated by the following: 


$$
\varepsilon_{\text {tool }}=\frac{S_{t o o l, 1}-S_{t o o l, 2}}{F\left(T_{T C, 1}\right)-F\left(T_{T C, 2}\right)}
$$

And in a similar manner to the formation of Equation (15), where the temperature of the microblackbody is assumed equal to the temperature of the surface:

$$
\varepsilon_{\text {tool }}=\varepsilon_{m B B} \frac{S_{t o o l, 1}-S_{t o o l, 2}}{S_{m B B, 1}-S_{m B B, 2}}
$$

These equations are summarized in Table 7 with the required assumptions for each technique.

Table 7: Summary of emissivity calculation methods utilizing thermocouple temperature $\left(T_{T C}\right)$,

\begin{tabular}{|c|c|c|}
\hline Equation & Equation \# & Required Assumptions \\
\hline$\epsilon_{\text {tool }}=S_{\text {tool }} / F\left(T_{T C}\right)$ & $(14)$ & $T_{\text {tool }}=T_{T C}$ \\
\hline$\epsilon_{\text {tool }}=S_{\text {tool }} / S_{m B B}$ & $(15)$ & $\begin{array}{l}T_{\text {tool }}=T_{m B B} \\
\varepsilon_{m B B}=1\end{array}$ \\
\hline $\begin{array}{l}\epsilon_{m B B}=S_{m B B} / F\left(T_{T C}\right) \\
\epsilon_{\text {tool }}=\epsilon_{m B B} * S_{\text {tool }} / S_{m B B}\end{array}$ & $(15),(16)$ & $\begin{array}{l}T_{\text {tool }}=T_{m B B}=T_{T C} \\
\varepsilon_{m B B} \neq 1\end{array}$ \\
\hline$\epsilon_{\text {tool }}=\left(S_{\text {tool, } 1}-S_{\text {tool }, 2}\right) /\left(F\left(T_{T C, 1}\right)-F\left(T_{T C, 2}\right)\right)$ & (17) & $\begin{array}{l}\Delta \varepsilon_{m B B}=\Delta \varepsilon_{\text {tool }}=0 \\
T_{\text {tool }}=T_{T C}\end{array}$ \\
\hline$\epsilon_{\text {tool }}=\left(S_{\text {tool, } 1}-S_{\text {tool }, 2}\right) /\left(S_{m B B, 1}-S_{m B B, 2}\right)$ & $(18)$ & $\begin{array}{l}\Delta \varepsilon_{m B B}=\Delta \varepsilon_{\text {tool }}=0 \\
T_{\text {tool }}=T_{m B B} \quad \varepsilon_{m B B}=1\end{array}$ \\
\hline$\epsilon_{\text {tool }}=\epsilon_{m B B^{*}}\left(S_{\text {tool, } 1}-S_{\text {tool, },}\right) /\left(S_{m B B, 1}-S_{m B B, 2}\right)$ & $(16),(18)$ & $\begin{array}{l}\Delta \varepsilon_{\mu B B}=\Delta \varepsilon_{\text {tool }}=0 \\
T_{\text {tool }}=T_{m B B}=T_{T C}, \quad \varepsilon_{m B B} \neq 1\end{array}$ \\
\hline
\end{tabular}
camera signal in the tool ROI $\left(S_{\text {tool }}\right)$, and camera signal from the micro-blackbody ROI $\left(S_{m B B}\right)$

\subsection{Emissivity Measurement Results}

A total of five cutting inserts were measured, with resulting tool emissivity calculated based on the available data given in Table 8 . Some of the insert numbers 9k and 8 included microblackbodies, whereas others did not, and insert numbers $9 \mathrm{k}, 1 \mathrm{k}$, and $2 \mathrm{k}$ had measurements taken at two temperature values. Note that the row for Equation (16) gives the calculated microblackbody emissivity $\left(\varepsilon_{m B B}\right)$ based on $T_{T C}$, which is used to evaluate Equations (15) and (18) assuming $\varepsilon_{m B B} \neq 1$. 
Table 8: Resulting emissivity values for five tungsten carbide cutting inserts

\begin{tabular}{|c|c|c|c|c|c|c|c|c|}
\hline \multirow{2}{*}{$\begin{array}{r}\text { Insert Number : } \\
\text { Thermocouple, } \mathbf{T}_{\mathrm{TC}}\left[{ }^{\circ} \mathrm{C}\right]:\end{array}$} & \multicolumn{2}{|c|}{ 9k } & 8 & \multicolumn{2}{|c|}{$1 \mathbf{k}$} & \multicolumn{2}{|c|}{$2 \mathbf{k}$} & \multirow{2}{*}{$\begin{array}{c}3 \\
544 \\
\end{array}$} \\
\hline & 446 & 545 & 544 & 446 & 548 & 445 & 545 & \\
\hline Equation (14) & 0.689 & 0.655 & 0.741 & 0.799 & 0.771 & 0.850 & 0.800 & 0.773 \\
\hline Equation $(15), \epsilon_{\mathrm{mBB}}=1$ & 0.808 & 0.814 & 0.850 & $*$ & $*$ & $*$ & $*$ & $*$ \\
\hline Equation $(16),\left(\epsilon_{\mathrm{mBB}}\right)$ & 0.853 & 0.805 & 0.870 & $*$ & $*$ & * & $*$ & $*$ \\
\hline Equation $(15) \&(16), \epsilon_{\mathrm{mBB}} \neq 1$ & 0.689 & 0.655 & 0.739 & $*$ & $*$ & $*$ & $*$ & $*$ \\
\hline Equation (17) & \multicolumn{2}{|c|}{0.641} & $* *$ & \multicolumn{2}{|c|}{0.760} & \multicolumn{2}{|c|}{0.782} & $* *$ \\
\hline Equation $(18), \epsilon_{\mathrm{mBB}}=1$ & \multicolumn{2}{|c|}{0.817} & $* *$ & \multicolumn{2}{|c|}{$*$} & \multicolumn{2}{|c|}{$*$} & $* *$ \\
\hline Equation $(16) \&(18), \epsilon_{\mathrm{mBB}} \neq 1$ & 0.697 & 0.658 & $* *$ & \multicolumn{2}{|c|}{$*$} & \multicolumn{2}{|c|}{$*$} & $* *$ \\
\hline
\end{tabular}

As previously mentioned, it is assumed the micro-blackbody gives a more precise and accurate emissivity measurement than the thermocouple, and emissivity values calculated using Equations (15) and (18) are therefore more accurate since they rely on the micro-blackbody measurement. This is evidenced in Table 8. Emissivity values which used Equation (14) and relied on $T_{T C}$ ranged from 0.655 to 0.850 , whereas those that used Equation (18) and relied on the microblackbody ranged from 0.808 to 0.850 . Though only one insert (9k) was measured at two temperatures and had a micro-blackbody, the assumptions required to use Equation (18) (the two temperature method) are assumed to be more accurate than Equation (15) since subtracting signal values may remove any erroneously incurred offset. From here forth in this document, an emissivity of 0.822 is assumed, being the mean of the four measurements made using Equations (15) and (18).

Though more accurate than the thermocouple, the micro-blackbodies are expensive and time consuming to fabricate. Placing the thermocouple nearer the micro-blackbody on the exposed face of the insert, and ensuring good thermal contact will ensure more comparable temperature values. Future efforts will test a new method of thermocouple placement alongside microblackbodies, and test correlation between emissivity measurements depending on their respective values. Given good correlation, it is hoped that better thermocouple placement may reduce the need for micro-blackbodies.

\section{TRUE TEMPERATURE MEASUREMENT}

Once the calibration, linearization, PSF, and emissivity of an object are determined, the true temperature of a measured object may be determined. The following steps are taken to obtain and prepare the measured signal, $S_{\text {meas}}$ : 
1.) Measure the object with the infrared camera using the same in-camera NUC used in all previous steps, same optics, integration time, etc. and collect camera signal in units [DL]. Obtain a 100 frame e-plate measurement immediately afterward.

2.) Take the frame average $\left(\mu_{\mathrm{F}}\right)$ of the e-plate measurement and subtract it from each frame of the DL video pixel-by-pixel to obtain frames in units $[\Delta \mathrm{DL}]$.

3.) Linearize each pixel of each frame using Equations (6.2)-(6.3) to obtain frames in units [a.u.].

4.) Deblur the image using the PSF by doing the following:

a) Mirror the edge of each frame by 41 pixels (the width of the PSF, i.e., make the frame wider by 82 pixels)

b) Deconvolve the PSF from the mirrored image using the blind deconvolution algorithm and the measured PSF as a seed value

c) Extract the un-windowed region from the deconvolved frame. This is the measured signal, $S_{\text {meas }}$.

Finally, the measurement equation is used to convert $S_{\text {meas }}$ into true temperature, $T_{\text {true }}$ :

$$
S_{\text {meas }}=\varepsilon \cdot F\left(T_{\text {true }}\right)+(1-\varepsilon) \cdot F\left(T_{\text {amb }}\right)
$$

Here, $F$ is the Sakuma-Hattori calibration function given in Equation (7.2). $T_{a m b}$ is temperature of the ambient surroundings reflected off the measured surface. It is typically taken as $20{ }^{\circ} \mathrm{C}$, though for the calibration example in this document, and the relatively high emissivities, $T_{a m b}$ accounts for less than $1 \Delta \mathrm{DL}$ signal and is therefore negligible for this example calibration. True temperature is evaluated by inverting Equation (19) on a pixel-by-pixel, frame by frame basis:

$$
T_{\text {true }}=F^{-1}\left(\frac{S_{\text {meas }}-(1-\varepsilon) \cdot F\left(T_{\text {amb }}\right)}{\varepsilon}\right)
$$

Recall that $\mathrm{F}^{-1}$ is calculated using Equation (7.3) for each pixel. Figure 18 shows the results of converting an example frame to true temperature using the measured emissivity value of $\epsilon=0.822$. The example is from an orthogonal cutting test using a tungsten-carbide grooving insert, oxidized in the thermal vise described in Section 4, and cutting a Ti-6Al-4V workpiece at $90 \mathrm{~m} / \mathrm{min}$ surface speed and $0.225 \mathrm{~mm}$ per revolution feed (or $0.225 \mathrm{~mm}$ depth of cut). Note the nonlinear conversion from signal to temperature due to the nonlinear calibration equation $F$. 

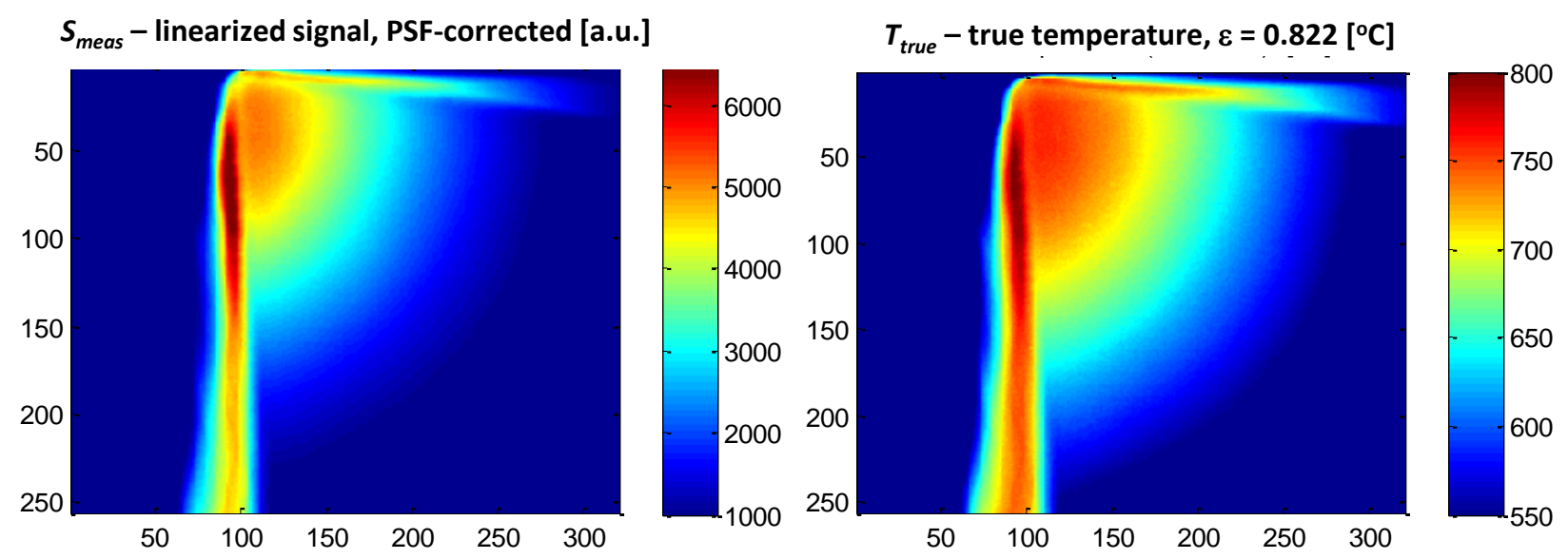

Figure 18: Conversion of a linearized, PSF-corrected infrared frame (left) to a true temperature frame (right) taken during machining experiments with the MOTS camera. Field of view of the image is approximately $0.87 \mathrm{~mm}$ vertical by $1.09 \mathrm{~mm}$ horizontal.

\section{DISCUSSION}

At this point, all steps in the process to convert infrared measurements into true temperature measurements have been given. However, a video consisting of a series of true temperature images is not the final measurement. Once a true temperature frame or set of frames is created, then the real measurement result is based on however the measurand is defined. In addition, final calculation of a measurement uncertainty value would depend on how the measurand is defined.

For example, the measurand may be defined as the true, physical peak temperature on the imaged surface (e.g., the tool face in Figure 18). However, this value may not be directly measurable due to the size-of-source effects that include sub-scenel averaging and blurring due to PSF $[1,2,23]$. Sub-scenel averaging describes how a pixel value results from an integrated radiance over the scenel area, and any radiating feature smaller than the scenel is lost in this integration. The effect is greater for surface regions of high temperature or emissivity gradients, but may be neglected for very uniform surfaces. Figure 19 gives a simulated example of a hot spot whose temperature is reduced through sub-scenel averaging and PSF blurring. While the effect of PSF blur may be corrected using the method developed in Section 3, no method is known to correct for sub-scenel averaging. 


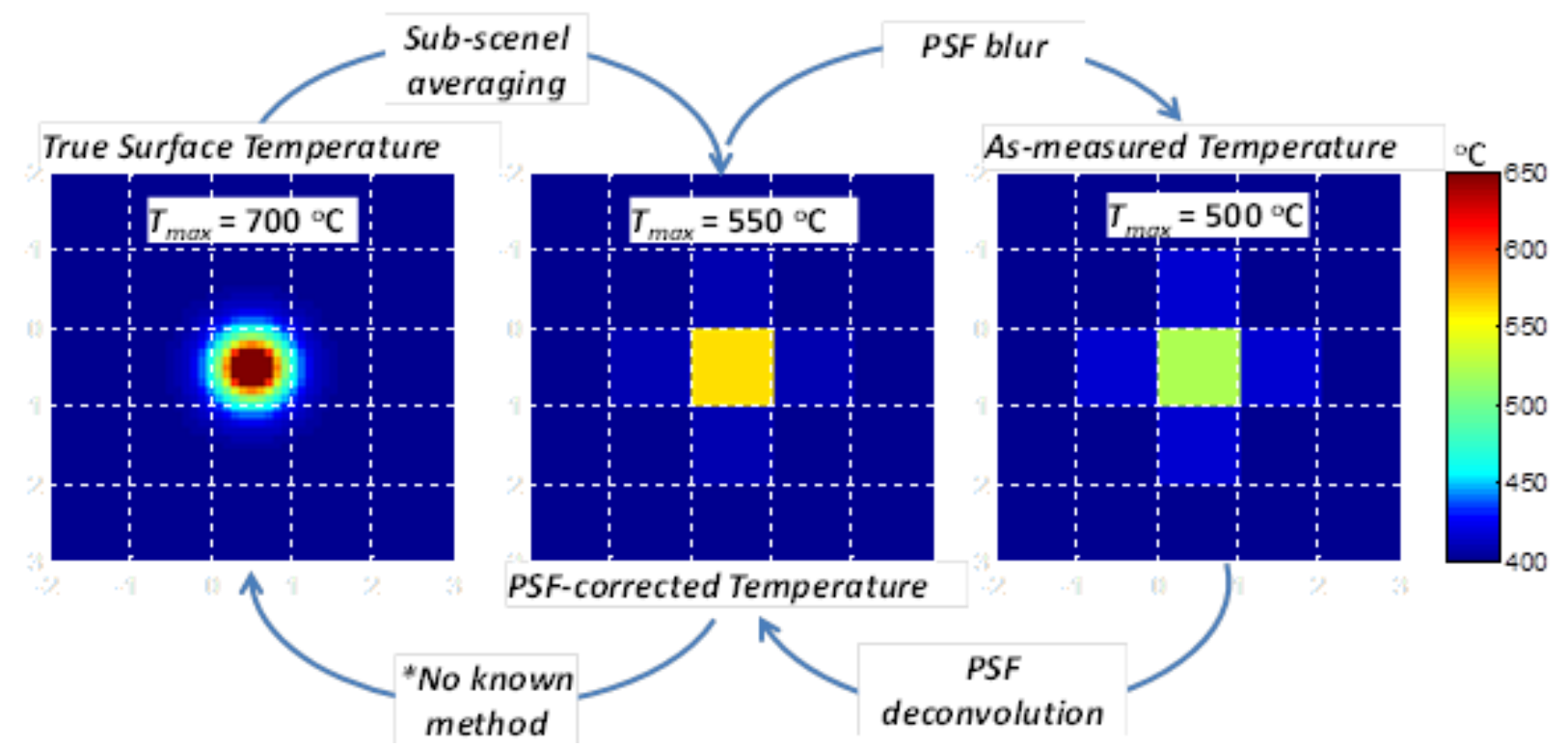

Figure 19: Size of source effects on an example measurement where the source is smaller than a scenel. No method is known to correct for sub-scenel averaging.

Temperature error due to sub-scenel averaging increases with the local temperature and emissivity gradients (or more simply, the apparent temperature gradient). However, for apparently uniform regions, such as the peak temperatures on the tool face in Figure 18, it is unlikely that local hot or cold spots exist that are smaller than a scenel size. In addition, if apparent temperature is uniform, the effects of PSF blurring is minimized. However, it is still recommended to correct for PSF blur since a methodology is known.

If the measurand is defined as the peak pixel value of the true temperature, no further steps are necessary beyond those mentioned in this document other than locating the peak pixel value. However, one might consider defining the measurand as the peak true temperature value of an average of $N$ pixels, since uncertainty of an average is inversely proportional to the root of $\mathrm{N}$. However, this also incurs greater error if the local apparent temperature near the peak is nonuniform. If the measurand is defined as the the peak pixel value of the true temperature on the object, and the object is moving, it may be necessary to include edge detection or object tracking algorithms to locate the object in each frame.

\section{CONCLUSIONS}

This document outlines the steps used to calibrate an infrared thermal camera and convert collected images into true temperature measurements. This process was developed based on previous work by the authors, and with the intent to reduce measurement uncertainty from known sources and more easily and accurately evaluate measurement uncertainty overall. The steps developed include: 1) linearization, 2) blackbody calibration, 3) PSF measurement, 
4) emissivity measurement, and 5) object temperature measurement. While linearization, calibration and object temperature measurement processes (i.e., converting a measured signal to true temperature using an emissivity) are available from thermal camera manufacturers as internal operations of the camera or camera operating software, these algorithms are proprietary, therefore calculating a measurement uncertainty is difficult. Also, previous work by the authors determined that accurate emissivity measurement and PSF measurement were key to reducing overall measurement uncertainty. Therefore, new processes for PSF and emissivity measurement were developed, tested, and are disseminated in this document, with recommendations for future improvements. 


\section{REFERENCES}

[1] B. Lane, E. Whitenton, V. Madhavan, A. Donmez, Uncertainty of temperature measurements by infrared thermography for metal cutting applications, Metrologia. 50 (2013) 637-653.

[2] E.P. Whitenton, High-speed dual-spectrum imaging for the measurement of metal cutting temperatures, National Institute of Standards and Technology, Gaithersburg, MD, 2010. http://www.nist.gov/customcf/get_pdf.cfm?pub_id=904301.

[3] W. Minkina, S. Dudzik, Infrared Thermography: Errors and Uncertainties, Wiley Online Library, 2009.

[4] G. Eppeldauer, G. Sauter, J. Gardner, Uncertainties of spectral responsivity measurements, Opt. Radiat. Meas. Based Detect. Stand. (2009) 134.

[5] J.L. Gardner, Uncertainties in interpolated spectral data, J. Res. - Natl. Inst. Stand. Technol. 108 (2003) 69-78.

[6] B.W. Mangum, G.T. Furukawa, K.G. Kreider, C.W. Meyer, D.C. Ripple, G.F. Strouse, et al., The Kelvin and temperature measurements, J. Res. Natl. Inst. Stand. Technol. 106 (2001) 105.

[7] P. Saunders, D. White, Propagation of uncertainty due to non-linearity in radiation thermometers, Int. J. Thermophys. 28 (2007) 2098-2110.

[8] G.C. Holst, Testing and evaluation of infrared imaging systems, JCD Pub.; SPIE Press, Winter Park, Fla.; Bellingham, Wash., 2008.

[9] F. Sakuma, S. Hattori, Establishing a practical temperature standard by using a narrow-band radiation thermometer with a silicon detector, in: Temp. Its Meas. Control Sci. Ind., AIP, New York, 1982: pp. 421-427.

[10] P. Saunders, Propagation of uncertainty for non-linear calibration equations with an application in radiation thermometry, Metrologia. 40 (2003) 93.

[11] P. Saunders, D.R. White, Physical basis of interpolation equations for radiation thermometry, Metrologia. 40 (2003) 195.

[12] M. Estribeau, P. Magnan, Fast MTF measurement of CMOS imagers using ISO 12333 slanted-edge methodology, in: Proc. SPIE, St. Etienne, France, 2003: pp. 243-252.

[13] ISO 12233:2014, Photography - Electronic still-picture cameras - Resolution measurements, ISO, Geneva, Switzerland, n.d.

[14] A.P. Tzannes, J.M. Mooney, Measurement of the modulation transfer function of infrared cameras, Opt. Eng. 34 (1995) 1808-1817. doi:10.1117/12.203133.

[15] E.W. Marchand, Derivation of the Point Spread Function from the Line Spread Function, J. Opt. Soc. Am. 54 (1964) 915-919. doi:10.1364/JOSA.54.000915.

[16] S. Deans, Radon and Abel Transforms, in: A. Poularikas (Ed.), Transforms Appl. Handb. Second Ed., CRC 2000. http://www.crcnetbase.com/doi/abs/10.1201/9781420036756.ch8 (accessed January 7, 2014).

[17] E.H.B. Smith, PSF estimation by gradient descent fit to the ESF, in: 2006: p. 60590E60590E-9. doi:10.1117/12.643071.

[18] H.J. Caulfield, Handbook of optical holography, Academic Press, New York, 1979.

[19] B.N. Taylor, C.E. Kuyatt, Guidelines for evaluating and expressing the uncertainty of NIST measurement results, 1994. 
[20] R.P. Madding, Emissivity measurement and temperature correction accuracy considerations, in: D.H.; S. John R.; LeMieux (Ed.), Proc. SPIE, Orlando, FL, 1999: pp. 393-401.

[21] C. S. Williams, Discussion of the theories of cavity-type sources of radiant energy, J. Opt. Soc. Am. 51 (1961) 564-571. doi:10.1364/JOSA.51.000564.

[22] R.W. Ivester, Tool temperatures in orthogonal cutting of alloyed titanium, in: Trans NAMRI-SME, Corvallis, OR, 2011: pp. 253-9.

[23] J.C. Heigel, E.P. Whitenton, The effects of integration time and size-of-source on the temperature measurement of segmented chip formation using infrared thermography, in: Proc. MSEC09, American Society of Mechanical Engineers, West Lafayette, IN, 2009: pp. 499-508. 


\section{APPENDIX A. NOTATION AND NOMENCLATURE FOR IMAGE STATISTICS}

The measured signal data from the thermal camera is converted and stored as a 3-dimensional array. Here, the first indices $(i, j)$ represent the pixels in each frame, and the third index $k$ represents a frame number. Statistics may be taken across a frame or along a pixel. The frame mean $\mu_{F}$ is the mean of the 256 pixels x 320 pixels in a single frame. During blackbody measurements, 100 frames are taken at a time. The pixel mean $\mu_{P}(i, j)$ is the average value of each pixel over the 100 frames, and results in a 256 pixel x 320 pixel array or frame.

$$
\mu_{F}=\frac{1}{256} \frac{1}{320} \sum_{j=1}^{320} \sum_{i=1}^{256} S(i, j, k=1) \quad \mu_{P}(i, j)=\frac{1}{100} \sum_{k=1}^{100} S(i, j, k)
$$

The frame mean of the pixel mean the gives a single value which is the approximate signal level at a calibration point:

$$
\mu_{F}\left(\mu_{P}\right)=\sum_{j=1}^{320} \sum_{i=1}^{256} \frac{\mu_{P}(i, j)}{256 \cdot 320}
$$

A measure of non-uniformity in a calibration frame is made by calculating the frame noise $\boldsymbol{\sigma}_{\mathbf{F}}$ which is the standard deviation of the $320 \times 256$ pixels in a single frame. A measure of the pixelto-pixel nonuniformity of a calibration frame is the frame noise of the pixel mean of a calibration frame:

$$
\sigma_{F}\left(\mu_{P}\right)=\sqrt{\sum_{j=1}^{320} \sum_{i=1}^{256} \frac{\left(\mu_{P}(i, j)-\mu_{F}\left(\mu_{P}(i, j)\right)\right)^{2}}{256 \cdot 320}}
$$

During a blackbody or e-plate measurement, each pixel experiences fluctuation from frame to frame over the 100-frame measurement. Taking the standard deviation of this fluctuation gives the pixel noise.

$$
\sigma_{P}(i, j)=\sqrt{\frac{1}{100} \sum_{k=1}^{100}\left(S(i, j, k)-\mu_{P}(i, j)\right)^{2}}
$$

Since each pixel has an associated pixel noise, a single-value measure of all the pixel noise is the frame mean of pixel noise

$$
\mu_{F}\left(\sigma_{P}\right)=\sum_{j=1}^{320} \sum_{i=1}^{256} \frac{\sigma_{P}(i, j)}{256 \cdot 320}
$$

A measure of how uniform the pixel noise is over the FPA is the frame noise of the pixel noise: 


$$
\sigma_{F}\left(\sigma_{p}\right)=\sqrt{\sum_{j=1}^{320} \sum_{i=1}^{256} \frac{\left(\sigma_{P}(i, j)-\mu_{F}\left(\sigma_{P}(i, j)\right)^{2}\right.}{256 \cdot 320}}
$$

Note that a calibration frame is the difference between a pixel mean of a blackbody measurement and pixel mean of an e-plate measurement:

$$
S(i, j)=\mu_{P, b b}(i, j)-\mu_{P, e p}(i, j)
$$

This is important to keep in mind for calculating the pixel-by-pixel calibration uncertainty which will require a sum of squares of the standard error of the means for the component of uncertainty due to electronic noise. However, in this document and in Table 1, statistics for the calibration frames are only meant as a representation of their mean values or a general measure of the FPA noise. 


\section{APPENDIX B. SPECTRAL RESPONSIVITY AND SAKUMA-HATTORI EQUATION RELATIONSHIPS}

Saunders [11] developed a relationship between spectral responsivity of a detector and the expected coefficients A, B, and C of the form of the Sakuma-Hattori equation given in equation (7.2). Spectral responsivity of the detector is characterized by the central wavelength, $\lambda_{0}$, and the variance, $\sigma^{2}$ of the spectral band. For a rectangular-shaped (or uniformly distributed) spectral responsivity, $\sigma^{2}=\Delta \lambda^{2} / 12$, where $\Delta \lambda$ is the bandwidth. Given a known spectral responsivity such as that of the MOTS camera in Figure 2, the central wavelength and variance are given as follows:

$$
\begin{gathered}
\lambda_{0}=\frac{\int \lambda w_{\lambda} d \lambda}{\int w_{\lambda} d \lambda} \\
\sigma^{2}=\frac{\int\left(\lambda-\lambda_{0}\right) d \lambda}{\int w_{\lambda} d \lambda}
\end{gathered}
$$

For sufficiently narrow bandwidths $\left(\sigma / \lambda_{0} \ll 1\right)$, these are related to the Sakuma-Hattori coefficients through the following:

$$
\begin{gathered}
\lambda_{0}=\frac{c_{2} A}{c_{2}-12 B} \\
\sigma^{2}=\frac{2 c_{2} A^{2} B}{\left(c_{2}-12 B\right)^{2}}
\end{gathered}
$$

With these equations, either an equivalent spectral responsivity may be deduced from a calibration using the Sakuma-Hattori equation, or, a signal response to a blackbody may be deduced from a known responsivity:

$$
\begin{gathered}
A=\lambda_{0}\left(1-6\left(\frac{\sigma}{\lambda}\right)^{2}\right) \\
B=\frac{c_{2}}{2}\left(\frac{\sigma}{\lambda}\right)^{2} \\
C=\int_{0}^{\infty} \frac{w_{\lambda}}{\lambda^{5}} d \lambda=\frac{a}{\lambda_{0}^{5}}
\end{gathered}
$$

The coefficient $a$ depends on the particular detector, electronics amplification, pixel size, optics geometry and transmission [8], similar to the coefficient $\alpha$ given in Equation (5) in Section 2.2. 


\section{APPENDIX C. CALIBRATION DATA STRUCTURE AND FUNCTIONS}

All the linearization and calibration data and statistics results are stored in the 'calibration structure' in Matlab. This structure will be fed to other functions to calculate PSF, emissivity, or convert video files. For example, to access the 'A' Sakuma-Hattori coefficient for the $(45,100)$ pixel, type: Cal_Mit_0550.SH.A(45,100).

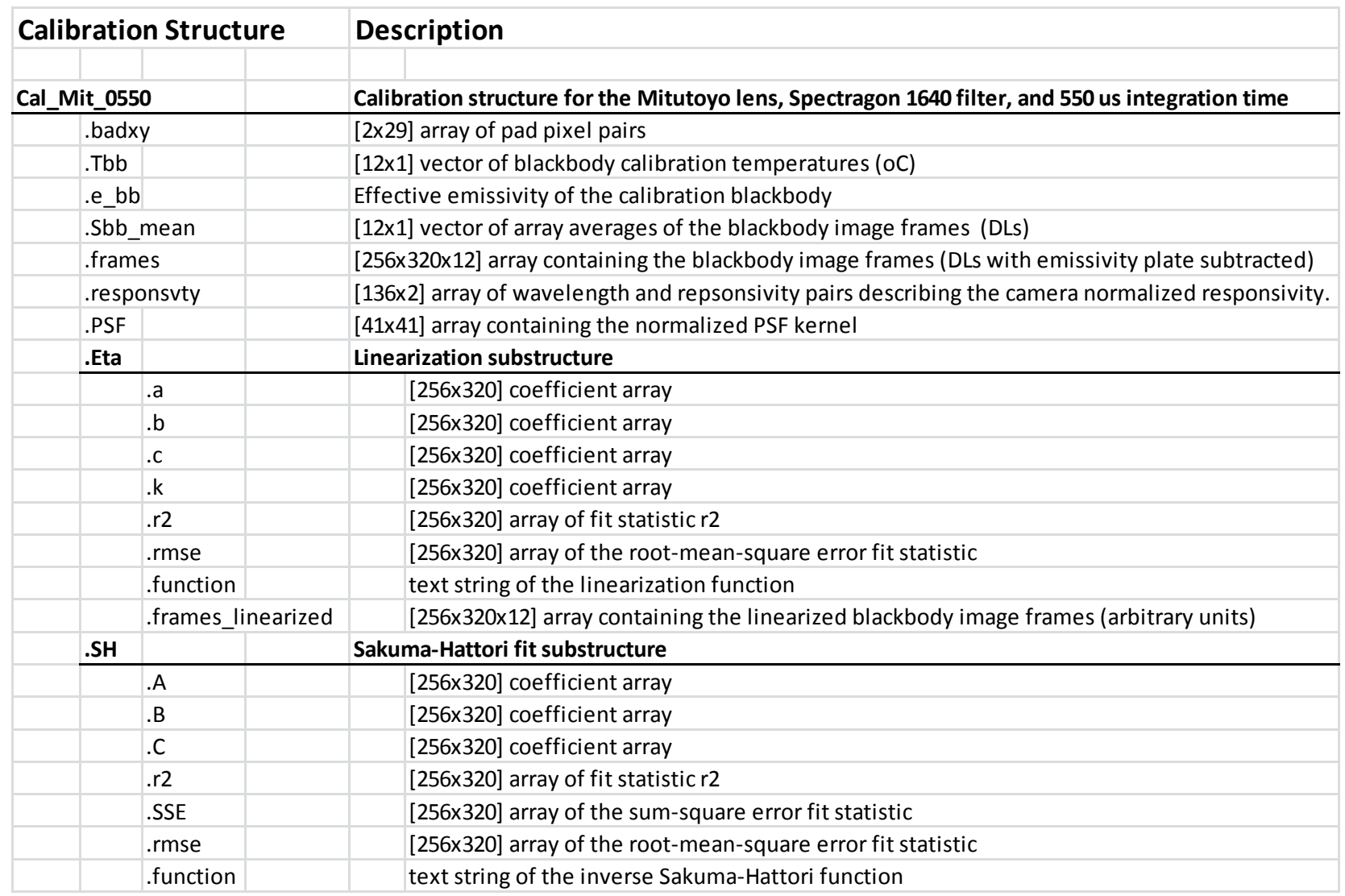

